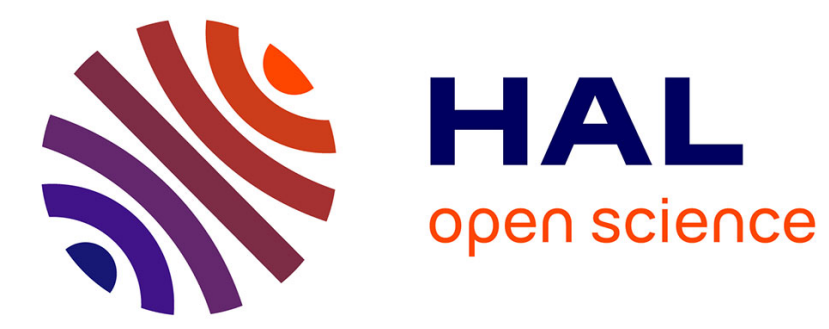

\title{
THE BORDIGA SURFACE AS CRITICAL LOCUS FOR 3-VIEW RECONSTRUCTIONS
}

\author{
Marina Bertolini, Roberto Notari, Christina Turrini
}

\section{To cite this version:}

Marina Bertolini, Roberto Notari, Christina Turrini. THE BORDIGA SURFACE AS CRITICAL LOCUS FOR 3-VIEW RECONSTRUCTIONS. MEGA 2017 - International Conference on Effective Methods in Algebraic Geometry, Jun 2017, Nice, France. hal-01829503

\section{HAL Id: hal-01829503 https://hal.inria.fr/hal-01829503}

Submitted on 4 Jul 2018

HAL is a multi-disciplinary open access archive for the deposit and dissemination of scientific research documents, whether they are published or not. The documents may come from teaching and research institutions in France or abroad, or from public or private research centers.
L'archive ouverte pluridisciplinaire $\mathbf{H A L}$, est destinée au dépôt et à la diffusion de documents scientifiques de niveau recherche, publiés ou non, émanant des établissements d'enseignement et de recherche français ou étrangers, des laboratoires publics ou privés. 


\title{
THE BORDIGA SURFACE AS CRITICAL LOCUS FOR 3-VIEW RECONSTRUCTIONS
}

\author{
MARINA BERTOLINI, ROBERTO NOTARI, AND CRISTINA TURRINI
}

\begin{abstract}
In Computer Vision, images of dynamic or segmented scenes are modeled as linear projections from $\mathbb{P}^{k}$ to $\mathbb{P}^{2}$. The reconstruction problem consists in recovering the position of the projected objects and the projections themselves from their images, after identifying many enough correspondences between the images. A critical locus for the reconstruction problem is a variety in $\mathbb{P}^{k}$ containing the objects for which the reconstruction fails. In this paper, we deal with projections both of points from $\mathbb{P}^{4} \rightarrow \mathbb{P}^{2}$ and of lines from $\mathbb{P}^{3} \rightarrow \mathbb{P}^{2}$. In both cases, we consider 3 projections, minimal number for a uniquely determined reconstruction. In the case of projections of points, we declinate the Grassmann tensors introduced in [21] in our context, and we use them to compute the equations of the critical locus. Then, given the ideal that defines this locus, we prove that, in the general case, it defines a Bordiga surface, or a scheme in the same irreducible component of the associated Hilbert scheme. Furthermore, we prove that every Bordiga surface is actually the critical locus for the reconstruction for suitable projections. In the case of projections of lines, we compute the defining ideal of the critical locus, that is the union of $3 \alpha$-planes and a line congruence of bi-degree $(3,6)$ and sectional genus 5 in the Grassmannian $\mathbb{G}(1,3) \subset \mathbb{P}^{5}$. This last surface is biregular to a Bordiga surface [40]. We use this fact to link the two reconstruction problems by showing how to compute the projections of one of the two settings, given the projections of the other one. The link is effective, in the sense that we describe an algorithm to compute the projection matrices.
\end{abstract}

\section{INTRODUCTION}

Linear projections from $\mathbb{P}^{3}$ to $\mathbb{P}^{2}$ are the natural geometric model for pictures of static three-dimensional scenes taken from pinhole cameras. Similarly linear projections from $\mathbb{P}^{k}$ to $\mathbb{P}^{2}$, with $k \geq 3$, arise when images of particular dynamic and segmented scenes are considered ([39, 21, 28, 38, 22]).

Given multiple images of an unknown scene, taken from unknown cameras, the reconstruction of the positions of cameras and scene points is a classical problem in Computer Vision, which has been generalized as well in the setting of higher dimensional projective spaces.

Sufficiently many images and sufficiently many sets of corresponding points in the given images should in principle allow for a successful projective reconstruction. Anyway, there exist sets of points, in the ambient space $\mathbb{P}^{k}$, for which the projective reconstruction fails. These configurations of points are called critical, which means that there exist other non projectively equivalent sets of points and cameras that give the same images in the view planes.

Critical loci for projections from $\mathbb{P}^{3}$ to $\mathbb{P}^{2}$ have been studied by many authors. Among the many papers on the subject, we recall $[13,27,29,19,24,20,35,2]$. In the case of projections from higher dimensional $\mathbb{P}^{k}$ to the projective plane $\mathbb{P}^{2}$, when $k \geq 4$, critical loci were described in [11] in the case of one view, and in $[7,3,6]$ in the case of multiple views.

Date: March 7, 2018.

2010 Mathematics Subject Classification. 14J25, 14M12, 14M15, $14 \mathrm{~N} 05$.

Key words and phrases. Bordiga surface, Line congruences in Grassmannians, Projective reconstruction in Computer Vision, Multiview Geometry, Critical configurations or loci.

The authors are members of GNSAGA of INdAM. 
In this paper we focus on the case of three projections from $\mathbb{P}^{4}$ to $\mathbb{P}^{2}$, that is the first nonclassical case, since three views is the minimum number which allows us to reconstruct the scene, when the scene points are general (in a sense which will be clear later). Our purpose is to get a scheme-theoretical description of the critical locus. The critical locus comes out to be a classical surface in $\mathbb{P}^{4}$, the so-called Bordiga surface ([12]). The approach used here to obtain the polynomials that generate the ideal of the critical locus is different from the one followed in [7]: we use the Grassmann tensor introduced in [21]. In [2], a first seminal case of this idea has been applied to two projections from $\mathbb{P}^{2}$ to $\mathbb{P}^{1}$, while in [10] this approach is used to study the critical locus when it is a hypersurface. The construction of the critical locus given in [7] allowed the set-theoretical description of it, while the one given here through the Grassmann tensor allows us to compute the generators of the ideal of the critical locus and its first syzygy module, so giving a scheme-theoretical description of the critical locus itself. In more details, the ideal is minimally generated by 4 degree 3 forms that are the maximal minors of a $4 \times 3$ matrix with linear entries. From the Hilbert-Burch Theorem, it follows that the critical locus is a determinantal variety of codimension 2 and degree 6 in $\mathbb{P}^{4}$, and so it belongs to the irreducible component of the Hilbert scheme containing Bordiga surfaces.

A very natural question arising as a consequence of the above results is whether every Bordiga surface in $\mathbb{P}^{4}$ is the critical locus of suitable projections. To give a positive answer to this question, we heavily use the geometry of Bordiga surfaces.

We recall that a Bordiga surface $S$ is the blow-up of $\mathbb{P}^{2}$ at 10 general points, embedded in $\mathbb{P}^{4}$ via the complete linear system of the quartics through the 10 points. $S$ contains exactly 10 lines, corresponding to the base points of the linear system. The ideal of the 10 points is determinantal, too. The $5 \times 4$ matrix of linear forms whose maximal minors are the generators of the ideal of the 10 points in $\mathbb{P}^{2}$ is strongly related to the matrix that presents the surface $S$ in $\mathbb{P}^{4}$. We use such a relation to prove that the generators of the first syzygy module of the ideal of $S$ can be chosen in a very particular form. Finally, we use this particular presentation to prove that every Bordiga surface is the critical locus for suitable projections, that we explicitly construct.

Quite surprisingly, the Bordiga surface is linked to another classical problem in Computer Vision: reconstruction using lines. Given an unknown scene in $\mathbb{P}^{3}$, consisting of a set of lines (i.e. a subset of the Grassmannian $\mathbb{G}(1,3)$ ), and taken multiple images of this scene, in which corresponding lines in the different views are identified, the goal is the reconstruction of the scene. The reconstruction using lines is particularly significant in the real word, since images of lines can be more easily and accurately detected and tracked than points [30]. A classical result of Buchanan and Maybank shows that the critical locus for reconstruction in $\mathbb{P}^{3}$ using lines is essentially a line congruence of bidegree $(3,6)$ and sectional genus 5 in $\mathbb{G}(1,3)$. This congruence admits a biregular model in $\mathbb{P}^{4}$ which is a Bordiga surface [40]. Furthermore, Maybank proves that every Bordiga surface can be obtained in this way, i.e. as model in $\mathbb{P}^{4}$ of a congruence in $\mathbb{G}(1,3)$ which arises as the critical locus for the reconstruction using lines. As a consequence of this, we have more deeply investigated, also from an algebraic point of view, the relations between the two critical loci. To do this we have computed the generators of the ideal of the critical congruence, obtaining its scheme-theoretical description, and proved that the whole critical locus in $\mathbb{G}(1,3)$ is the union of the quoted congruence with three $\alpha$-planes corresponding to the lines through the centers of projection.

Finally we have explicitly described how to link the projections from $\mathbb{P}^{3}$ to $\mathbb{P}^{2}$ used in the reconstruction using lines, with the ones from $\mathbb{P}^{4}$ to $\mathbb{P}^{2}$ used in the reconstruction using points, which give rise to biregular critical loci, and conversely. This provides a connection between the two reconstructions, a priori independent each other.

The plan of the paper is as follows. In sections 2 and 3 we recall the geometric constructions of the Bordiga surface and of the $(3,6)$ line congruence respectively and, for 
both of them, we give the algebraic translation of their constructions. Moreover in section 3 we present some results on the $(3,6)$ line congruence we are not able to quote. Section 4 is devoted to recall the main facts on multiview geometry in higher dimension, in particular the description of the Grassmann tensor. In section 5 we prove that the generators of the ideal of the critical locus for 3 projections from $\mathbb{P}^{4}$ to $\mathbb{P}^{2}$ can be deduced via the appropriate Grassmann tensor and that this locus is actually a Bordiga surface. Moreover we prove also that the converse holds, i.e. every Bordiga surface $S$ is the critical locus of suitable linear projections. In section 6 we provide the definition of critical locus for reconstruction using lines, we compute the defining ideal of such a critical locus and finally we show that the $(3,6)$ congruence is the main component of this locus. Section 7 gives a bridge between the two above problems of criticality, by explicitly computing the equations of the maps between the two critical loci. From the maps we are able to compute the projections of one of the two settings given the projections of the other one. In section 8 we provide a Singular session in which we compute a degree 10 arithmetically Gorenstein curve contained in a given $(3,6)$ line congruence. We need this curve to make effective the algorithm in section 7 .

\section{On the Bordiga SURFACE}

In this section, we recall the construction of the Bordiga surface and some properties of this surface we'll use later on in the paper. For more information on this surface, see $[12],[16]$.

Let $\mathbb{P}^{2}=\operatorname{Proj}\left(S=\mathbb{C}\left[z_{0}, z_{1}, z_{2}\right]\right)$ be the projective space of dimension 2 over the complex ground field $\mathbb{C}$, and let $p_{1}, \ldots, p_{10} \in \mathbb{P}^{2}$ be ten general points. Let $\widetilde{\mathbb{P}}^{2} \stackrel{\pi}{\longrightarrow} \mathbb{P}^{2}$ be the blowup of $\mathbb{P}^{2}$ in $p_{1}, \ldots, p_{10}$. The linear system $\left|4 \pi^{*} L-E_{1}-\cdots-E_{10}\right|$, where $L$ is the line divisor in $\mathbb{P}^{2}$ and $E_{i}$ is the exceptional divisor associated to $p_{i}, i=1, \ldots, 10$, embeds $\widetilde{\mathbb{P}}^{2}$ in $\mathbb{P}^{4}=\operatorname{Proj}\left(R=\mathbb{C}\left[y_{0}, \ldots, y_{4}\right]\right)$, and becomes the hyperplane divisor $H$ of the image. If $\varphi: \widetilde{\mathbb{P}}^{2} \hookrightarrow \mathbb{P}^{4}$ is the embedding, $B=\varphi\left(\widetilde{\mathbb{P}}^{2}\right)$ is named Bordiga surface. $B$ is a smooth surface of degree 6 and sectional genus 3. $\varphi$ maps each $E_{i}$ onto a line, and moreover $B$ contains exactly these 10 lines. It is possible to prove that these lines are pairwise skew and that no three of them are contained in a 3 -dimensional linear space. The canonical divisor of $B$ is $K_{B}=\left|-3 \pi^{*} L+E_{1}+\cdots+E_{10}\right|$. By adjunction, it is possible to prove that the image of a plane cubic through 9 among $p_{1}, \ldots, p_{10}$ is a plane cubic in $B$, as well. Moreover, the plane containing it and the exceptional line associated to the 10th point are skew linear spaces. Finally, the image of a line containing no point among $p_{1}, \ldots, p_{10}$ is a rational normal quartic curve $\Gamma$ contained in $B$.

Now, we give the algebraic translation of the previous geometric constructions.

Because of the generality assumption, $Z=\left\{p_{1}, \ldots, p_{10}\right\}$ has Hilbert function $h_{Z}(j)=$ $\min \left\{\left(\begin{array}{c}j+2 \\ 2\end{array}\right), 10\right\}$ for $j \geq 0$, and so $I_{Z}$, defining ideal of $Z$ in $S$, is generated by 5 forms $g_{0}, \ldots, g_{4}$ of degree 4 . As $Z$ is a codimension 2 arithmetically Cohen-Macaulay (ACM, for short) closed subscheme of $\mathbb{P}^{2}$, the ideal $I_{Z}$ is determinantal and its minimal free resolution is

$$
0 \rightarrow S^{4}(-5) \stackrel{N_{Z}}{\longrightarrow} S^{5}(-4) \rightarrow I_{Z} \rightarrow 0 .
$$

The forms $g_{0}, \ldots, g_{4}$ are a basis of the complete linear system of quartic curves through $p_{1}, \ldots, p_{10}$. If we define $\varphi: R \rightarrow S$ as $\varphi\left(y_{i}\right)=g_{i}, i=0, \ldots, 4$, then $I_{B}=\operatorname{ker}(\varphi)$ is the defining ideal of the Bordiga surface $B=\varphi\left(\widetilde{\mathbb{P}}^{2}\right) . I_{B}$ is determinantal too, and its minimal free resolution is

$$
0 \rightarrow R^{3}(-4) \stackrel{N_{B}}{\longrightarrow} R^{4}(-3) \rightarrow I_{B} \rightarrow 0
$$

This proves that $B$ is a codimension $2, \mathrm{ACM}$ closed scheme in $\mathbb{P}^{4}$ with Hilbert polynomial $p_{B}(t)=\left(6 t^{2}+2 t+2\right) / 2$. From [18], it follows that 
Proposition 2.1. The Bordiga surface is the general element of the irreducible component of the Hilbert scheme $\mathcal{H} i b_{p_{B}(t)}\left(\mathbb{P}^{4}\right)$ containing the codimension 2, ACM closed subschemes of $\mathbb{P}^{4}$ with Hilbert polynomial $p_{B}(t)$.

From the point of view of liaison theory, the Bordiga surface is linked to a degree 3 rational normal scroll. In fact, let $S$ be the surface complete intersection two general cubic hypersurfaces containing $B$. Then, by standard argument from liaison theory, the defining ideal of the residual surface $B^{\prime}$ has the following minimal free resolution

$$
0 \rightarrow R^{2}(-3) \rightarrow R^{3}(-2) \rightarrow I_{B^{\prime}} \rightarrow 0
$$

and so $B^{\prime}$ is a degree 3 rational normal scroll, as claimed. Moreover, the intersection between $B$ and $B^{\prime}$ is an arithmetically Gorenstein curve of degree 8 and socle degree 3 , as its minimal free resolution is

$$
0 \rightarrow R(-6) \rightarrow \underset{R^{3}(-4)}{\oplus} \rightarrow \underset{R^{2}(-3)}{\oplus} \rightarrow I_{B \cap B^{\prime}} \rightarrow 0
$$

computed by mapping cone from the short exact sequence

$$
0 \rightarrow I_{S} \rightarrow I_{B} \oplus I_{B^{\prime}} \rightarrow I_{B \cap B^{\prime}} \rightarrow 0 .
$$

Such a curve is the image in $B$ of a curve in the linear system $\left|7 \pi^{*} L-2 E_{1}-\cdots-2 E_{10}\right|=$ $\left|-K_{B}+H\right|$ and so the curve is a twisted anticanonical divisor on $B$ (see, [25] for generalities on such divisors). Such a linear system will play a role in next sections.

By adjunction ([32], pp.48-49), one can prove that the matrices $N_{Z}$ and $N_{B}$ are related each other by

$$
N_{B}\left(\begin{array}{c}
z_{0} \\
z_{1} \\
z_{2}
\end{array}\right)=N_{Z}^{T}\left(\begin{array}{c}
y_{0} \\
\vdots \\
y_{4}
\end{array}\right)
$$

If $p \in \mathbb{P}^{2} \backslash Z$, then $\operatorname{rank}\left(N_{Z} \otimes \mathbb{C}(p)\right)=4$ as (1) remains exact after tensorization by $\mathbb{C}(p)$, residue field of $p$. So $p$ is mapped to the point in $\mathbb{P}^{4}$ whose homogeneous coordinates solve the linear system

$$
\left(N_{Z} \otimes \mathbb{C}(p)\right)^{T}\left(\begin{array}{c}
y_{0} \\
\vdots \\
y_{4}
\end{array}\right)=\mathbf{0} .
$$

If $p \in Z$, then $\operatorname{rank}\left(N_{Z} \otimes \mathbb{C}(p)\right)=3$, and so the solutions of (4) are the points of the exceptional line $E_{P}$. Moreover, if $p \in Z$, then $I_{Z}: I_{p}$ has a unique generator in degree 3 , the defining form $f_{C}$ of the plane cubic curve $C$ through $Z \backslash\{p\}$. For every line $L \ni p$, we have that $l f_{C} \in\left\langle g_{0}, \ldots, g_{4}\right\rangle$, with $l \in \mathbb{C}\left[z_{0}, z_{1}, z_{2}\right]_{1}$ defining $L$, and so $l f_{c}=$ $a_{0}(l) g_{0}+\cdots+a_{4}(l) g_{4}$ for suitable $a_{i}(l) \in \mathbb{C}$. Then, the hyperplane $a_{0}(l) y_{0}+\ldots a_{4}(l) y_{4}=0$ contains $\varphi(C)$. As there are two linearly independent lines in $\mathbb{P}^{2}$ containing $p$, we have that $\varphi(C)$ is contained in a dimension 2 linear space, as well. Hence, $\varphi(C)$ is a plane cubic, because $I_{B}$ is generated in degree 3 . Now we want to explain why the above plane containing $\varphi(C)$ is skew with $E_{p}$. In fact, the solutions of (4) define the sub-linear system of plane quartics through $p_{1}, \ldots, p_{10}$, singular at $p$. As the quartics defined by $l f_{C}$ are smooth at $p$, then they are not in the sub-linear system above, and so the intersection of the two linear spaces is empty.

Now, if $L \subset \mathbb{P}^{2}$ is a general line, we can assume that $L \cap Z=\emptyset$. Let $p, q \in L$ be two distinct points and let $M_{L}=\left(\begin{array}{cc}z_{0 p} & z_{0 q} \\ z_{1 p} & z_{1 q} \\ z_{2 p} & z_{2 q}\end{array}\right)$ be the rank 2 matrix whose columns are homogeneous coordinates of the points $p, q$. Then $N_{B} M_{L}$ is a $4 \times 2$ matrix of generic rank 
2 of linear forms in $R$, and so its maximal minors define a rational normal curve $\Gamma$ of degree 4 contained in $B$.

\section{On the Line CONGRUENCE OF BI-DEgRee $(3,6)$ AND SECTIONAl Genus 5}

In this section, we recall the construction and some properties of the line congruence $K$ of bi-degree $(3,6)$ and sectional genus 5 in the Grassmannian $\mathbb{G}(1,3)$ of lines in $\mathbb{P}^{3}$, following [40], [1]. Furthermore, we present three properties of $K$ for which we are not able to quote a reference.

As in section 2 , let $\widetilde{\mathbb{P}}^{2}$ be the blow-up of $\mathbb{P}^{2}$ at 10 general points $p_{1}, \ldots, p_{10}$. The linear system $\left|7 \pi^{*} L-2 E_{1}-\cdots-2 E_{10}\right|$ on $\widetilde{\mathbb{P}}^{2}$ embeds $\widetilde{\mathbb{P}}^{2}$ in $\mathbb{G}(1,3) \subset \mathbb{P}^{5}=\operatorname{Proj}(T=$ $\left.\mathbb{C}\left[x_{0}, \ldots, x_{5}\right]\right)$. For a suitable choice of the coordinates, $\mathbb{G}(1,3)=V\left(x_{0} x_{5}-x_{1} x_{4}+x_{2} x_{3}\right)$. Let $\psi: \widetilde{\mathbb{P}}^{2} \rightarrow \mathbb{G}(1,3)$ be such an embedding, and let $K=\psi\left(\widetilde{\mathbb{P}}^{2}\right)$ be its image. Then, $K$ is a line congruence of bi-degree $(3,6)$ and sectional genus 5 . The canonical divisor is $K_{K}=\left|-3 \pi^{*} L+E_{1}+\cdots+E_{10}\right|$ while the hyperplane divisor is $H=\left|7 \pi^{*} L-2 E_{1}-\cdots-2 E_{10}\right|$. Moreover, the converse holds true, that is to say, every such a surface is the embedding of $\widetilde{\mathbb{P}}^{2}$ with $\left|7 \pi^{*} L-2 E_{1}-\cdots-2 E_{10}\right|$. In $\mathbb{G}(1,3)$, the ideal sheaf $\mathcal{I}_{K \mid \mathbb{G}(1,3)}$ is generated in degree 3 and it holds

$$
0 \rightarrow \mathcal{O}_{\mathbb{G}(1,3)}^{5} \rightarrow \mathcal{E}_{2}(1)^{3} \rightarrow \mathcal{I}_{K \mid \mathbb{G}(1,3)}(3) \rightarrow 0
$$

where $\mathcal{E}_{2}$ is the rank 2 vector bundle on $\mathbb{G}(1,3)$ coming from the universal exact sequence

$$
0 \rightarrow \mathcal{E}_{1} \rightarrow H^{0}\left(\mathcal{O}_{\mathbb{P}^{3}}(1)\right) \otimes \mathcal{O}_{\mathbb{G}(1,3)} \rightarrow \mathcal{E}_{2}(1) \rightarrow 0 .
$$

The first result we are not able to quote concerns the shape of the minimal free resolution of the defining ideal $I_{K}$ of $K$ in $T$.

Proposition 3.1. The minimal free resolution of $I_{K}$ has the shape

$$
0 \rightarrow T^{5}(-5) \rightarrow T^{12}(-4) \rightarrow \underset{T^{7}(-3)}{\oplus} \rightarrow I_{K} \rightarrow 0 .
$$

Proof. From (5), we get the resolution of $I_{K \mid \mathbb{G}(1,3)}$ over $T_{\mathbb{G}}=T /\left\langle x_{0} x_{5}-x_{1} x_{4}+x_{2} x_{3}\right\rangle$, that is periodic, and it is

$$
\cdots \rightarrow T_{\mathbb{G}}^{12}(-5) \rightarrow T_{\mathbb{G}}^{12}(-4) \rightarrow T_{\mathbb{G}}^{7}(-3) \rightarrow I_{K \mid \mathbb{G}(1,3)} \rightarrow 0
$$

as $H^{0}\left(\mathcal{E}_{2}(1)\right)=H^{0}\left(\mathcal{O}_{\mathbb{P}^{3}}(1)\right)$. By [34], the minimal free resolution of $I_{K}$ has the shape

$$
0 \rightarrow T^{5}(-5) \rightarrow T^{12}(-4) \rightarrow \underset{T^{7}(-3)}{\oplus} \rightarrow I_{K} \rightarrow 0
$$

as claimed, where the degree 2 generator of $I_{K}$ is the defining form of $\mathbb{G}(1,3)$.

In particular, $K$ is $\mathrm{ACM}$ with Hilbert polynomial $p_{K}(t)=\left(9 t^{2}+t+2\right) / 2$. A result similar to Proposition 2.1 holds.

Proposition 3.2. The line congruence of bi-degree $(3,6)$ and sectional genus 5 is the general element of the irreducible component of the Hilbert scheme $\mathcal{H} i b_{p_{K}(t)}\left(\mathbb{P}^{5}\right)$ containing the codimension $3, A C M$ closed subschemes of $\mathbb{P}^{5}$ with Hilbert polynomial $p_{K}(t)$ and resolution (6).

Now, we go back to the geometric description of $K$.

As $\widetilde{\mathbb{P}}^{2}$ is embedded both in $\mathbb{P}^{4}$ as a Bordiga surface $B$, and in $\mathbb{G}(1,3)$ as a suitable line congruence $K$, it is natural to look for maps from $\mathbb{P}^{4}$ to $\mathbb{G}(1,3)$ that transform $B$ into $K$. 
In [40], it is proved that the map $\theta$ that makes commutative the diagram



is associated to the linear system $\left|\mathcal{O}_{\mathbb{P}^{4}}(2) \backslash \Gamma\right|$ where $\Gamma \subset B$ is a degree 4 rational normal curve in $\mathbb{P}^{4}$, and so $\Gamma$ is the image in $B$ of a line in $\mathbb{P}^{2}$. The map $\theta$ contracts lines that are bi-secant to $\Gamma$ and so $\theta$ is an embedding of $\mathbb{P}^{4} \backslash F$ where $F$ is the secant variety to $\Gamma$, a degree 3 hypersurface. Moreover, $\theta$ maps $F$ to a Veronese surface $V$.

Now, we can prove the second result we are not able to quote.

Theorem 3.1. The line congruence $K$ of bi-degree $(3,6)$ and sectional genus 5 in $\mathbb{G}(1,3)$ is Gorenstein linked to a Veronese surface $V$ by an arithmetically Gorenstein surface $S$ of degree 13 and socle degree 4.

Proof. The map $\theta^{-1}$ is defined on $K \backslash C$ where $C=K \cap V$ and $V$ is the contraction of the secant variety to $\Gamma$ where $\theta$ is not an embedding. The curve $C$ is in the linear system $\left|10 \pi^{*} L-3 E_{1}-\cdots-3 E_{10}\right|=\left|-K_{K}+H\right|$ and so $C$ is a twisted anticanonical divisor. By ([25], Lemma 5.4), $C$ is arithmetically Gorenstein in $\mathbb{P}^{5}$, and its minimal free resolution can be easily constructed by self-duality, because the minimal free resolution of $I_{V}$ is a subcomplex of the resolution of $I_{C}$. Then, we have

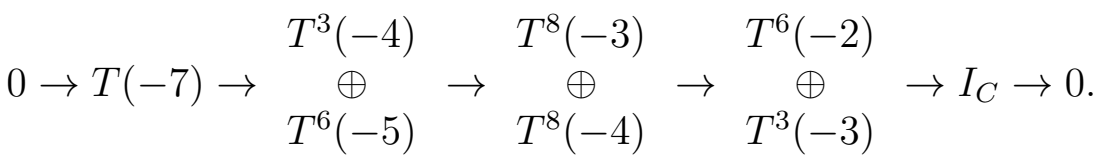

Let $S=K \cup V$. Then, we have the short exact sequence

$$
0 \rightarrow I_{S} \rightarrow I_{K} \oplus I_{V} \rightarrow I_{C} \rightarrow 0
$$

from which we get the shape of the minimal free resolution of $I_{S}$ :

$$
0 \rightarrow T(-7) \rightarrow \underset{\oplus}{T(-5)} \rightarrow \begin{gathered}
T^{4}(-4) \\
T^{4}(-3)
\end{gathered} \rightarrow I_{S} \rightarrow 0
$$

and so we get that $S$ is arithmetically Gorenstein of degree 13 and socle degree 4, as claimed. Moreover, $K$ is algebraically linked to $V$ by $S$ and the linkage is geometric, because both $K$ and $V$ are irreducible varieties, and so they have no irreducible component in common.

Conversely, let $V \subset \mathbb{G}(1,3)$ be a Veronese surface, and let $S$ be an arithmetically Gorenstein surface of degree 13 and socle degree 4 containing $V$ and contained in the Grassmannian $\mathbb{G}(1,3)$. Then, the Hilbert function of the Artinian reduction of $S$ is $(1,3,5,3,1)$, and so its minimal free resolution is (8). Hence, the residual variety $K$ has minimal free resolution (6) and so $K$ is a line congruence of bi-degree $(3,6)$ and sectional genus 5, as claimed.

Remark 3.1. As the shape of the resolution of $I_{C}$ allows cancellations, the general element of $\left|-K_{K}+H\right|$ is not the intersection of $K$ with a Veronese surface.

Now, we describe an algorithm, based on [31], to compute such a surface $S$, given a general line congruence $K$. It can be easily adapted to start from $V$. In last section 8, we implement it in Singular ([15]), as this explicit computation is needed in section 7, which is the algorithmic part of the paper.

Given a reduced Gröbner basis of $I_{K}$ with respect to the degree reverse lexicographic ordering (degrevlex, for short) in $T$, the initial ideal in $\left(I_{K}\right)$ is generated by $\left\langle x_{2} x_{3}, x_{0}^{3}, x_{0}^{2} x_{1}\right.$, $\left.x_{0} x_{1}^{2}, x_{1}^{3}, x_{0}^{2} x_{2}, x_{0} x_{1} x_{2}, x_{1}^{2} x_{2}\right\rangle$. With "general" line congruence, we mean that the generators of in $\left(I_{K}\right)$ are as large as possible w.r.t. the degrevlex ordering and the Hilbert function 
of $I_{K}$. Then, we write polynomials $p_{1}, \ldots, p_{5}$ whose coefficients are indeterminates, and whose initial terms are $x_{0}^{3}, x_{0}^{2} x_{1}, x_{0} x_{1}^{2}, x_{0}^{2} x_{2}, x_{1}^{4}$, respectively. Also in this case, we choose the largest monomial ideal compatible with the Hilbert function of $S$. We want to compute the indeterminate coefficients in such a way that $\left\{x_{0} x_{5}-x_{1} x_{4}+x_{2} x_{3}, p_{1}, p_{2}, p_{3}, p_{4}, p_{5}\right\}$ is a reduced Gröbner basis of $I_{S}$. At first, we reduce $p_{1}, \ldots, p_{5}$ w.r.t. the Gröbner basis of $I_{K}$ and we set equal to 0 their normal forms. This gives a linear system in the indeterminate coefficients. We compute the solutions of this system, and we substitute them in the polynomials $p_{1}, \ldots, p_{5}$. Then, we run the Buchberger algorithm on the input $\left\{x_{0} x_{5}-x_{1} x_{4}+x_{2} x_{3}, p_{1}, p_{2}, p_{3}, p_{4}, p_{5}\right\}$ and we set equal to zero their S-polynomials. We get a new set of equations, no more linear. It is possible to check, however, that the equations allow us to express all but two of the remaining variables as rational functions of the last two ones. More precisely, the two special variables are the coefficients of $x_{1}^{2} x_{2}, x_{0} x_{1} x_{2}$ in $p_{4}$. For a random choice of them, we compute the polynomials $p_{1}, \ldots, p_{5}$ and we get the arithmetically Gorenstein surface $S$ we are looking for. As a by-product, we have that such surfaces form a dimension 2 family, whose parameter space contains an open subset of an affine plane $\mathbb{A}^{2}$. This confirms that for every line in $\mathbb{P}^{2}$, containing no point among $p_{1}, \ldots, p_{10}$, we can construct a rational normal curve $\Gamma$ in the Bordiga surface $B \subset \mathbb{P}^{4}$ and a curve $C$ contained in $K \subset \mathbb{G}(1,3)$ where the map $\theta$ is not an embedding.

As last result, we describe a geometric property of $K$.

We proved in section 2 that a plane cubic curve containing 9 of the 10 points in $Z$ is mapped onto a plane cubic curve in $B$, as well. Now we prove that these plane curves are mapped by $\theta$ to plane curves again. Take, for example, an element in $\left|3 \pi^{*} L-E_{2}-\cdots-E_{10}\right|$. The degree of its image in $K$ is 3 and its genus, computed via adjunction, turns out to be 1 . So it is a plane curve in $K$, too. The plane containing this curve is an $\alpha$-plane in $\mathbb{G}(1,3)$. To compute the equations of the $\alpha$-plane, one can proceed as follows. Let $C_{i} \subset \mathbb{P}^{2}$ be the cubic curve not containing $p_{i}, i=1, \ldots, 10$, and let $\ell_{i j}$ be the line through $p_{i}, p_{j}$, with $i \neq j$. Then, $\ell_{i j} C_{i} C_{j}$ is a degree 7 plane curve singular at $p_{1}, \ldots, p_{10}$, and so it is a linear combination of a basis of the linear system. These linear combinations give the equations of the hyperplanes in $\mathbb{G}(1,3) \subset \mathbb{P}^{5}$ containing the image of $C_{i}$. If we select $p_{1}, p_{2}, p_{3}$ as distinguished points among $p_{1}, \ldots, p_{10}$, and we set $\alpha_{1}, \alpha_{2}, \alpha_{3}$ the planes containing the images of $C_{1}, C_{2}, C_{3}$, respectively, then each one of them intersects the other two ones, as the intersection point is image of the further intersection point of $C_{i}$ and $C_{j}$ other then the base points.

Now, we can prove the last result we are not able to quote.

Proposition 3.3. With the previous notations, let $Y$ be the union of the line congruence $K$ and the three planes $\alpha_{1}, \alpha_{2}, \alpha_{3}$. Then, $Y$ is an $A C M$ surface of bi-degree $(6,6)$ in $\mathbb{G}(1,3)$ and the minimal free resolution of its defining ideal is

$$
0 \rightarrow T^{3}(-6) \rightarrow \underset{\substack{T^{3}(-4) \\ T^{4}(-5)}}{\rightarrow} \underset{\substack{\oplus \\ T^{4}(-3)}}{\rightarrow} \rightarrow I_{Y} \rightarrow 0 .
$$

Proof. To prove the result, we use mainly mapping cone procedure. The starting point is the following short exact sequence

$$
0 \rightarrow I_{K \cap\left(\alpha_{1} \cup \alpha_{2}\right)} \rightarrow I_{K \cap \alpha_{1}} \oplus I_{K \cap \alpha_{2}} \rightarrow I_{K \cap \alpha_{1} \cap \alpha_{2}} \rightarrow 0 .
$$

As $K \cap \alpha_{i}$ is a degree 3 plane curve, the minimal free resolution of $I_{K \cap \alpha_{i}}$ is Koszul, with 3 generators in degree 1 and a further generator in degree 3 . On the other hand, $K \cap \alpha_{1} \cap \alpha_{2}$ is a point, and so also the minimal free resolution of $I_{K \cap \alpha_{1} \cap \alpha_{2}}$ is Koszul with 5 generators in degree 1. Furthermore, $K \cap\left(\alpha_{1} \cup \alpha_{2}\right)$ is the union of two plane cubic curves meeting at a point, and so there are two minimal generators in degree 3. 
Then, by mapping cone on the previous sequence, we get



Before going on, we construct the minimal free resolution of $I_{\alpha_{1} \cup \alpha_{2} \cup \alpha_{3}}$. We choose coordinates in $\mathbb{P}^{5}$ in such a way that $I_{\alpha_{1}}=\left\langle x_{0}, x_{1}, x_{2}\right\rangle, I_{\alpha_{2}}=\left\langle x_{0}, x_{3}, x_{4}\right\rangle, I_{\alpha_{3}}=\left\langle x_{1}, x_{3}, x_{5}\right\rangle$, so to let them intersect pairwise. Hence, $I_{\alpha_{1} \cup \alpha_{2} \cup \alpha_{3}}=\left\langle x_{0} x_{1}, x_{0} x_{3}, x_{0} x_{5}, x_{1} x_{3}, x_{1}, x_{4}, x_{2} x_{3}\right.$, $\left.x_{2} x_{4} x_{5}\right\rangle$, and its minimal free resolution has the shape

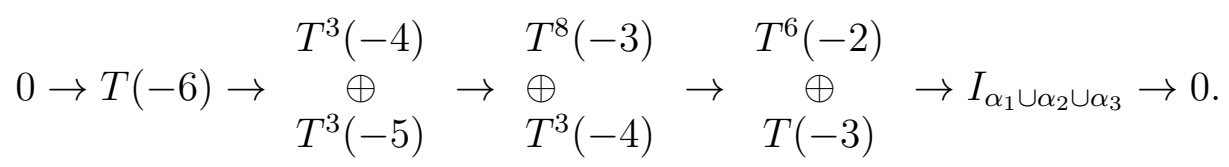

Now, we consider the short exact sequence

$$
0 \rightarrow I_{K \cap\left(\alpha_{1} \cup \alpha_{2} \cup \alpha_{3}\right)} \rightarrow I_{K \cap\left(\alpha_{1} \cup \alpha_{2}\right)} \oplus I_{K \cap \alpha_{3}} \rightarrow I_{K \cap\left(\alpha_{1} \cup \alpha_{2}\right) \cap \alpha_{3}} \rightarrow 0 .
$$

When we apply the mapping cone procedure, we consider that, by degree argument, the minimal free resolution of $I_{\alpha_{1} \cup \alpha_{2} \cup \alpha_{3}}$ is a sub-complex of the resolution of $I_{K \cap\left(\alpha_{1} \cup \alpha_{2} \cup \alpha_{3}\right)}$, and that $K \cap\left(\alpha_{1} \cup \alpha_{2}\right) \cap \alpha_{3}$ is a set of 2 points, and so its minimal free resolution is Koszul, too, with 4 generators in degree 1 and a generator in degree 2 . Then, we get

$$
0 \rightarrow T^{4}(-6) \rightarrow \underset{T^{12}(-5)}{\oplus} \rightarrow \underset{T^{12}(-4)}{\oplus} \rightarrow \underset{\substack{T^{3}(-4) \\ T^{4}(-3)}}{\rightarrow} \rightarrow I_{K \cap\left(\alpha_{1} \cup \alpha_{2} \cup \alpha_{3}\right)} \rightarrow 0 .
$$

Finally, from the short exact sequence

$$
0 \rightarrow I_{Y} \rightarrow I_{K} \oplus I_{\alpha_{1} \cup \alpha_{2} \cup \alpha_{3}} \rightarrow I_{K \cap\left(\alpha_{1} \cup \alpha_{2} \cup \alpha_{3}\right)} \rightarrow 0
$$

we get the result on the minimal free resolution of $I_{Y}$. From the minimal free resolution, it follows that $Y$ is ACM.

\section{On multiview Geometry for projections from $\mathbb{P}^{4}$ to $\mathbb{P}^{2}$}

In this section we fix notation and terminology and give a short overview of classical facts in Computer Vision related to the problem of projective reconstruction of scenes and cameras from multiple views.

Even if all the definitions can be given in full generality, we restrict ourselves to the case of projections from $\mathbb{P}^{4}$ to $\mathbb{P}^{2}$. In this context, a camera $P$ is a linear projection from $\mathbb{P}^{4}$ onto $\mathbb{P}^{2}$, from a line $C_{P}$, called center of projection. The target space $\mathbb{P}^{2}$ is called view. A scene is a set of points $\mathbf{X}_{i} \in \mathbb{P}^{4}$.

Using homogeneous coordinates in $\mathbb{P}^{4}$ and $\mathbb{P}^{2}$, we identify $P$ with a $3 \times 5$ matrix of maximal rank, defined up to a multiplicative constant. Hence $C_{P}$ comes out to be the right annihilator of $P$.

Let us consider a set of cameras $P_{j}: \mathbb{P}^{4} \backslash C_{P_{j}} \rightarrow \mathbb{P}^{2}$ projecting the same scene in $\mathbb{P}^{4}$ and the corresponding set of images in the different target planes. In this setting, proper linear subspaces (points or lines), $L_{i}, i=1 \ldots m$, of different views are said to be corresponding if there exists at least a point $\mathbf{X} \in \mathbb{P}^{4}$ such that $P_{i}(\mathbf{X}) \in L_{i}$ for all $i=1 \ldots m$.

In the context of multiple view geometry, the problem of projective reconstruction of a scene, given multiple images of it, is the following: given many enough scene points in $\mathbb{P}^{4}$ and identified a suitable number of corresponding subspaces on each image, one wants to get the projection matrices (up to projective transformations), i.e. the cameras, and the coordinates in $\mathbb{P}^{4}$ of the scene points.

Hartley and Schaffalitzky, [21], have constructed a set of multiview tensors, called Grassmann tensors, encoding the relations between sets of corresponding subspaces. We recall 
here the basic elements of their construction in the case of three projections from $\mathbb{P}^{4}$ to $\mathbb{P}^{2}$, which turns out to be the minimal number of views allowing projective reconstruction.

Consider three projections $P_{j}: \mathbb{P}^{4} \backslash C_{j} \rightarrow \mathbb{P}^{2}, j=1,2,3$, with centers $C_{1}, C_{2}, C_{3}$ in general position.

Let $\left\{L_{1}, L_{2}, L_{3}\right\}$ be three general linear subspaces (points or lines) of $\mathbb{P}^{2}$ of codimension $\alpha_{1}, \alpha_{2}, \alpha_{3}$, respectively. $\left(L_{1}, L_{2}, L_{3}\right)$ is a triple of corresponding subspaces if and only if $\left(P_{1}\right)^{-1}\left(L_{1}\right) \cap\left(P_{2}\right)^{-1}\left(L_{2}\right) \cap\left(P_{3}\right)^{-1}\left(L_{3}\right)$ is not empty. From the Grassmann formula, if $\sum \alpha_{j}=5, j=1,2,3$, the existence of points in the previous intersection gives a constrain which allows us to construct the Grassmann tensor. Hartley and Schaffalitzky call the triple $\left(\alpha_{1}, \alpha_{2}, \alpha_{3}\right)$ a profile for the reconstruction problem. In our situation the only possible profiles are: $\left(\alpha_{1}, \alpha_{2}, \alpha_{3}\right)=(2,2,1),(2,1,2)$ or $(1,2,2)$.

Let $\left\{L_{1}, L_{2}, L_{3}\right\}$ be three general linear subspaces of $\mathbb{P}^{2}$ as above and let $S_{j}$ be the maximal rank matrix of type $3 \times\left(3-\alpha_{j}\right)$ whose columns are a basis for $L_{j}, j=1,2,3$. By definition, if the $L_{j}$ 's are corresponding subspaces, there exists a point $\mathbf{X} \in \mathbb{P}^{k}$ such that $P_{j}(\mathbf{X}) \in L_{j}$ for $j=1,2,3$. In other words there exist three vectors $\mathbf{v}_{\mathbf{j}} \in \mathbb{C}^{3-\alpha_{j}} j=1,2,3$ such that:

$$
\left(\begin{array}{cccc}
S_{1} & 0 & 0 & P_{1} \\
0 & S_{2} & 0 & P_{2} \\
0 & 0 & S_{3} & P_{3}
\end{array}\right) \cdot\left(\begin{array}{c}
\mathbf{v}_{\mathbf{1}} \\
\mathbf{v}_{\mathbf{2}} \\
\mathbf{v}_{\mathbf{3}} \\
\mathbf{X}
\end{array}\right)=\left(\begin{array}{l}
0 \\
0 \\
0
\end{array}\right) .
$$

The existence of a non-trivial solution $\left(\mathbf{v}_{\mathbf{1}}, \mathbf{v}_{\mathbf{2}}, \mathbf{v}_{\mathbf{3}}, \mathbf{X}\right)$ of system (10) implies that the coefficient matrix has determinant zero, as it is square of order 9 in our case. This determinant can be thought of as a tri-linear form (tensor) in the Plücker coordinates of the spaces $L_{j}$. This tensor is the Grassmann tensor.

In the following we explicitly construct such a tensor for the profile $(2,2,1)$, others being similar. Now $L_{1}, L_{2}$ are points and $L_{3}$ is a line. We denote by $\left(x_{1}, x_{2}, x_{3}\right),\left(y_{1}, y_{2}, y_{3}\right)$ the homogeneous coordinates of $L_{1}$ and $L_{2}$, respectively, and by $\left(z_{1}, z_{2}, z_{3}\right)$ and $\left(w_{1}, w_{2}, w_{3}\right)$ the homogeneous coordinates of two points of $L_{3}$. In this case the matrix of the coefficients of the linear system (10) becomes:

$$
T_{L_{1}, L_{2}, L_{3}}^{P_{1}, P_{2}, P_{3}}=\left(\begin{array}{ccccccccc}
x_{1} & 0 & 0 & 0 & P_{1}[1,1] & P_{1}[1,2] & P_{1}[1,3] & P_{1}[1,4] & P_{1}[1,5] \\
x_{2} & 0 & 0 & 0 & P_{1}[2,1] & P_{1}[2,2] & P_{1}[2,3] & P_{1}[2,4] & P_{1}[2,5] \\
x_{3} & 0 & 0 & 0 & P_{1}[3,1] & P_{1}[3,2] & P_{1}[3,3] & P_{1}[3,4] & P_{1}[3,5] \\
0 & y_{1} & 0 & 0 & P_{2}[1,1] & P_{2}[1,2] & P_{2}[1,3] & P_{2}[1,4] & P_{2}[1,5] \\
0 & y_{2} & 0 & 0 & P_{2}[2,1] & P_{2}[2,2] & P_{2}[2,3] & P_{2}[2,4] & P_{2}[2,5] \\
0 & y_{3} & 0 & 0 & P_{2}[3,1] & P_{2}[3,2] & P_{2}[3,3] & P_{2}[3,4] & P_{2}[3,5] \\
0 & 0 & z_{1} & w_{1} & P_{3}[1,1] & P_{3}[1,2] & P_{3}[1,3] & P_{3}[1,4] & P_{3}[1,5] \\
0 & 0 & z_{2} & w_{2} & P_{3}[2,1] & P_{3}[2,2] & P_{3}[2,3] & P_{3}[2,4] & P_{3}[2,5] \\
0 & 0 & z_{3} & w_{3} & P_{3}[3,1] & P_{3}[3,2] & P_{3}[3,3] & P_{3}[3,4] & P_{3}[3,5]
\end{array}\right)
$$

where $P_{i}[j, k]$ denotes the element in position $(j, k)$ of the matrix $P_{i}$.

If $L_{1}, L_{2}$ and $L_{3}$ are corresponding spaces then the linear system

$$
T_{L_{1}, L_{2}, L_{3}}^{P_{1}, P_{2}, P_{3}}\left(\begin{array}{c}
\lambda \\
\mu \\
\alpha \\
\beta \\
\mathbf{X}
\end{array}\right)=\mathbf{0}
$$

has a non trivial solution, and so $\operatorname{det}\left(T_{L_{1}, L_{2}, L_{3}}^{P_{1}, P_{2}, P_{3}}\right)=0$.

The converse is true for general $L_{1}, L_{2}$ and $L_{3}$ since we are looking for a non trivial solution of (11) in which $\mathbf{X}$ is a point of $\mathbb{P}^{4}$ (i.e. $\mathbf{X} \neq \mathbf{0}$ ) and $\mathbf{X} \notin C_{1} \cup C_{2} \cup C_{3}$. 
In particular this happens if $L_{1} \notin P_{1}\left(C_{2} \cup C_{3}\right)$ and $L_{2} \notin P_{2}\left(C_{1} \cup C_{3}\right)$. Under this hypothesis, if $\operatorname{det}\left(T_{L_{1}, L_{2}, L_{3}}^{P_{1}, P_{2}, P_{3}}\right)=0$, then $L_{1}, L_{2}$ and $L_{3}$ are corresponding as the linear system (11) has a non trivial solution with $\mathbf{X}$ as required. Indeed the case $\mathbf{X}=\mathbf{0}$ doesn't occur, since, in the opposite case, either $L_{1}$ or $L_{2}$ are not points, or $L_{3}$ is not a line. Moreover $\mathbf{X} \notin C_{1} \cup C_{2} \cup C_{3}$, for the assumption.

In conclusion, for the chosen profile $(2,2,1)$, one sees that $\operatorname{det}\left(T_{L_{1}, L_{2}, L_{3}}^{P_{1}, P_{2}, P_{3}}\right)=0$ is indeed the tri-linear constraint between the coordinates $\mathbf{x}$ and $\mathbf{y}$ of the first and second view and the Plücker (i.e.dual) coordinates of the line $\langle\mathbf{z}, \mathbf{w}\rangle$ of the third view so to let them be corresponding.

\section{Critical loci for PRojective Reconstruction of POINTS AND their GEOMETRY}

As discussed in the previous section, folklore on the reconstruction problem says that sufficiently many views and sufficiently many sets of corresponding points in the given views should allow one a successful projective reconstruction. This is generally true, but even in the classical set-up of two projections from $\mathbb{P}^{3}$ to $\mathbb{P}^{2}$ one can have non projectively equivalent pairs of sets of scene points and of cameras that produce the same images in the view planes, thus preventing reconstruction. Such configurations and the loci they describe are referred to as critical. In [7], critical loci for projective reconstruction of camera centers and scene points from multiple views for projections from $\mathbb{P}^{k}$ to $\mathbb{P}^{2}$ have been introduced and studied. Here we shortly recall the basic definitions in the case in which we are interested in, i.e. three views from $\mathbb{P}^{4}$ to $\mathbb{P}^{2}$. Moreover we study the critical locus $\mathcal{X}$ for general projections. The non general case is under investigation, and some preliminary results are summarized in [9].

Definition 5.1. A set of points $\left\{\mathbf{X}_{j}\right\}, j=1, \ldots, N, N \gg 0$, in $\mathbb{P}^{4}$ is said to be a critical configuration for projective reconstruction from three views if there exist two collections of $3 \times 5$ full-rank projection matrices $P_{i}$ and $Q_{i}, i=1,2,3$, and a set of $N$ points $\left\{\mathbf{Y}_{j}\right\} \subset \mathbb{P}^{4}$, non-projectively equivalent to $\left\{\mathbf{X}_{j}\right\}$, such that, for all $i$ and $j, P_{i}\left(\mathbf{X}_{j}\right)=Q_{i}\left(\mathbf{Y}_{j}\right)$, up to homography in the image planes. The two sets $\left\{\mathbf{X}_{j}\right\}$ and $\left\{\mathbf{Y}_{j}\right\}$ are called conjugate critical configurations, with associated conjugate matrices $\left\{P_{i}\right\}$ and $\left\{Q_{i}\right\}$.

Remark 5.1. It can be proved that in the above definition $N \geq 7$ is enough.

The generators of the ideal of the critical locus $\mathcal{X}$ can be obtained by making use of the Grassmann tensor introduced in the previous section.

Indeed, the Grassmann tensor $\mathcal{T}^{P_{1}, P_{2}, P_{3}}$ encodes the algebraic relations between corresponding subspaces in the different views of the projections $P_{1}, P_{2}, P_{3}$. Hence by definition of critical set, if $\left\{\mathbf{X}_{j}, \mathbf{Y}_{j}\right\}$ are conjugate critical configurations, then, for each $j$, the projections $P_{1}\left(\mathbf{X}_{j}\right), P_{2}\left(\mathbf{X}_{j}\right)$ and $P_{3}\left(\mathbf{X}_{j}\right)$ are corresponding points not only for the projections $P_{1}, P_{2}, P_{3}$, but for the projections $Q_{1}, Q_{2}, Q_{3}$, too.

Following the construction of the previous section, we first choose the profile $(2,2,1)$.

If $L_{1}^{\prime}, L_{2}^{\prime}, L_{3}^{\prime}$ is a triple of corresponding spaces for the projections $Q_{1}, Q_{2}, Q_{3}$, where $L_{1}^{\prime}, L_{2}^{\prime}$ are points with homogeneous coordinates $L_{1}^{\prime}=\left(x_{1}^{\prime}, x_{2}^{\prime}, x_{3}^{\prime}\right)^{T}, L_{2}^{\prime}=\left(y_{1}^{\prime}, y_{2}^{\prime}, y_{3}^{\prime}\right)^{T}$ in the first two views respectively, and $L_{3}^{\prime}$ is the line spanned by $\mathbf{z}=\left(z_{1}^{\prime}, z_{2}^{\prime}, z_{3}^{\prime}\right)$ and $\mathbf{w}=\left(w_{1}^{\prime}, w_{2}^{\prime}, w_{3}^{\prime}\right)$ in the third view, the trilinear relation between $L_{1}^{\prime}, L_{2}^{\prime}, L_{3}^{\prime}$ is given by the vanishing of $\operatorname{det}\left(T_{L_{1}^{\prime}, L_{2}^{\prime}, L_{3}^{\prime}}^{Q_{1}, Q_{2}, Q_{3}}\right)$, where

$$
T_{L_{1}^{\prime}, L_{2}^{\prime}, L_{3}^{\prime}}^{Q_{1}, Q_{2}, Q_{3}}=\left(\begin{array}{ccccc}
L_{1}^{\prime} & 0 & 0 & 0 & Q_{1} \\
0 & L_{2}^{\prime} & 0 & 0 & Q_{2} \\
0 & 0 & \mathbf{z} & \mathbf{w} & Q_{3}
\end{array}\right)
$$

Considering as corresponding spaces $L_{1}^{\prime}=P_{1}(\mathbf{X}), L_{2}^{\prime}=P_{2}(\mathbf{X})$ and any line $L_{3}^{\prime}$ passing through $P_{3}(\mathbf{X})$, with $\mathbf{X}$ any point in the critical locus, one gets that the determinant of 
the matrix

$$
M^{\prime}=\left(\begin{array}{ccccc}
P_{1}(\mathbf{X}) & 0 & 0 & 0 & Q_{1} \\
0 & P_{2}(\mathbf{X}) & 0 & 0 & Q_{2} \\
0 & 0 & P_{3}(\mathbf{X}) & \mathbf{v} & Q_{3}
\end{array}\right)
$$

must vanish for every choice of $\mathbf{v}=(a, b, c)^{T}$.

This implies that all the cofactors of the elements $a, b, c$ in $M^{\prime}$ must vanish.

Since the above reasoning holds for all the possible profiles $(2,2,1),(2,1,2)$ or $(1,2,2)$, one sees that $\mathbf{X}$ is in the critical locus if and only if one has the vanishing of all the maximal minors of

$$
M=\left(\begin{array}{cccc}
P_{1}(\mathbf{X}) & 0 & 0 & Q_{1} \\
0 & P_{2}(\mathbf{X}) & 0 & Q_{2} \\
0 & 0 & P_{3}(\mathbf{X}) & Q_{3}
\end{array}\right)
$$

From the above discussion it follows that the maximal minors of $M$ generate the ideal of the critical locus $\mathcal{X}$, as $\mathbf{X}$ has to satisfy no other constraint.

Remark 5.2. In [7], the critical locus for a suitable number $n$ of projections from $\mathbb{P}^{k}$ to $\mathbb{P}^{2}$ has been introduced and studied in a more general context. If $k$ is even, the critical locus is a determinantal variety, defined by $3 n$ polynomials of degree $n$. In the particular case of three projections from $\mathbb{P}^{4}$ to $\mathbb{P}^{2}$, up to a constant, these polynomials are exactly the maximal minors of $M$ defined above.

Now we study the geometry of the critical locus $\mathcal{X}$ in a general situation, where general means that the greatest common divisor of the generators of the ideal is trivial.

Since the generators have a trivial greatest common divisor, by Hilbert-Burch Theorem ([17], Theorem 20.15), a free resolution of the ideal $I_{\mathcal{X}}$ they generate is

$$
0 \rightarrow \underset{R^{5}(-3)}{\oplus} \stackrel{R^{3}(-4)}{\longrightarrow} R^{9}(-3) \longrightarrow I_{\mathcal{X}} \rightarrow 0
$$

where $M$ is the above matrix.

By generality assumption, we can assume that rank $\left(\begin{array}{l}Q_{1} \\ Q_{2} \\ Q_{3}\end{array}\right)=5$. Hence, we can cancel the summand $R^{5}(-3)$ from the resolution, and we get the minimal free resolution

$$
0 \rightarrow R^{3}(-4) \stackrel{N_{\mathcal{X}}}{\longrightarrow} R^{4}(-3) \longrightarrow I_{\mathcal{X}} \rightarrow 0 .
$$

Let us assume that a non-vanishing minor is given by the last 5 rows and columns in $M$. Then if we write $M$ as the following block matrix

$$
M=\left(\begin{array}{cc}
A & B \\
C & D
\end{array}\right)
$$

with $A$ of type $4 \times 3$, and $D$ invertible, we can reduce $M$ with elementary operations on rows and columns to the block matrix

$$
\left(\begin{array}{cc}
N_{\mathcal{X}} & 0 \\
0 & I_{5}
\end{array}\right)
$$

where $I_{5}$ is the $5 \times 5$ identity matrix, 0 are null matrices of suitable type, and

$$
N_{\mathcal{X}}=A-B D^{-1} C \text {. }
$$

Because of the way it is obtained, $N_{\mathcal{X}}$ has linear entries. As the elementary operations on the columns correspond to base changes in the free module $R^{3}(-4) \oplus R^{5}(-3)$ while the ones on the rows correspond to base changes in the free module $R^{9}(-3)$, the global effect is to construct a new set of 4 generators of the ideal $I_{\mathcal{X}}$ again as maximal minors of $N_{\mathcal{X}}$. Then, the critical locus $\mathcal{X}$ is a codimension 2 arithmetically Cohen-Macaulay scheme in 
$\mathbb{P}^{4}$ with Hilbert polynomial $p(t)=\left(6 t^{2}+2 t+2\right) / 2=p_{B}(t)$. From Proposition 2.1 , we have the following

Proposition 5.1. The general critical locus for projective reconstruction for three views from $\mathbb{P}^{4}$ to $\mathbb{P}^{2}$ is in the irreducible component of $\mathcal{H i l b}_{p_{B}(t)}\left(\mathbb{P}^{4}\right)$ containing the Bordiga surfaces.

It is then natural to characterize the locus filled by critical loci inside this irreducible component. Quite surprisingly, this locus coincides with the whole irreducible component. In fact, we have

Theorem 5.1. Let $B$ be a Bordiga surface. Then $B$ is a critical locus for the projective reconstruction from three views from $\mathbb{P}^{4}$ to $\mathbb{P}^{2}$, that is to say, there exist two couples of three projections $P_{1}, P_{2}, P_{3}$ and $Q_{1}, Q_{2}, Q_{3}$ from $\mathbb{P}^{4}$ to $\mathbb{P}^{2}$ such that the associated critical locus is $B$.

Proof. Let $Z \subset \mathbb{P}^{2}$ be a set of 10 points in uniform position and let $B$ be the associated Bordiga surface. $B$ is irreducible, of course. We choose a reference frame in $\mathbb{P}^{2}$ in such a way that the fundamental points $(1: 0: 0),(0: 1: 0),(0: 0: 1)$ are in $Z$. For this choice, from equation (3), we have that the columns of $N_{B}$ in resolution (2) define three lines in $\mathbb{P}^{4}$ that lie on the Bordiga surface $B$. We take these lines as centers of three projections $P_{1}, P_{2}, P_{3}$.

By performing elementary operations on the rows of $N_{B}=\left(n_{i j}\right)$, we can assume that $n_{41}=0$ and $n_{11}, n_{21}, n_{31}$ are linearly independent.

Now, we want to prove that $n_{42} \neq 0$. Assume by contradiction that $n_{42}=0$. Then, with easy computations, we have that $I_{B}$ is generated by

$$
n_{43} \operatorname{det}\left(N_{\widehat{14 ; 3}}\right), n_{43} \operatorname{det}\left(N_{\widehat{24 ; 3}}\right), n_{43} \operatorname{det}\left(N_{\widehat{34 ; 3}}\right), \operatorname{det}\left(N_{\hat{4}}\right)
$$

So,

$$
I_{B}=\left\langle n_{43}, \operatorname{det}\left(N_{\hat{4}}\right)\right\rangle \cap\left\langle\operatorname{det}\left(N_{\widehat{14 ; 3}}\right), \operatorname{det}\left(N_{\widehat{24 ; 3}}\right), \operatorname{det}\left(N_{\widehat{34 ; 3}}\right)\right\rangle
$$

where $\widehat{i j ; k}$ denotes the cancellation of the rows $i, j$ and of the column $k$ from $N_{B}$, while $\widehat{i}$ denotes the cancellation of the $i$-th row, and hence $B$ is not irreducible. In more details, $\left\langle n_{43}, \operatorname{det}\left(N_{\hat{4}}\right)\right\rangle$ defines a cubic surface in a $\mathbb{P}^{3} \operatorname{while}\left\langle\operatorname{det}\left(N_{\widehat{14 ; 3}}\right), \operatorname{det}\left(N_{\widehat{24 ; 3}}\right), \operatorname{det}\left(N_{\widehat{34 ; 3}}\right)\right\rangle$ defines a cone in $\mathbb{P}^{4}$ over a twisted cubic curve in a suitable $\mathbb{P}^{3}$. Finally, the two surfaces meet along a twisted cubic curve. Then, $n_{42} \neq 0$.

Analogously we get $n_{43} \neq 0$.

Performing other elementary operations on the rows of $N_{B}$, we can get $n_{32}=0$. In analogy to the previous case, $n_{33} \neq 0$ otherwise $B$ would be non irreducible, as only an element in the third row of $N_{B}$ is non-zero. Let $\mathbf{p}_{3}^{1} \mathbf{X}, \mathbf{p}_{3}^{2} \mathbf{X}, \mathbf{p}_{3}^{3} \mathbf{X}$ be a basis of the ideal generated by $n_{i 3}, i=1, \ldots, 4$. Moreover, let $E$ be the $4 \times 3$ matrix such that

$$
\left(\begin{array}{l}
n_{13} \\
n_{23} \\
n_{33} \\
n_{43}
\end{array}\right)=E\left(\begin{array}{l}
\mathbf{p}_{3}^{1} \mathbf{X} \\
\mathbf{p}_{3}^{2} \mathbf{X} \\
\mathbf{p}_{3}^{3} \mathbf{X}
\end{array}\right)
$$

As $n_{33} \neq 0$ and $n_{43} \neq 0$, the last two rows of $E$ are non-zero, as well.

Then, if we set

$$
A=\left(\begin{array}{ccc}
n_{11} & 0 & 0 \\
n_{21} & 0 & 0 \\
n_{31} & 0 & 0 \\
0 & n_{42} & 0
\end{array}\right) \quad \text { and } \quad C=\left(\begin{array}{ccc}
0 & n_{12} & 0 \\
0 & n_{22} & 0 \\
0 & 0 & \mathbf{p}_{3}^{1} \mathbf{X} \\
0 & 0 & \mathbf{p}_{3}^{2} \mathbf{X} \\
0 & 0 & \mathbf{p}_{3}^{3} \mathbf{X}
\end{array}\right)
$$


we have that $N_{B}=A-F C$ for

$$
F=\left(\begin{array}{cc|c}
-1 & 0 & \\
0 & -1 & \\
0 & 0 & -E \\
0 & 0 &
\end{array}\right)
$$

Let $D$ be any invertible matrix of order 5 and take $B=F D$. Then, the maximal minor of the matrix

$$
M=\left(\begin{array}{cc}
A & B \\
C & D
\end{array}\right)
$$

generate the same ideal $I_{B}$ that defines the Bordiga surface $B$ from which we started.

The matrices $P_{1} \mathbf{X}, P_{2} \mathbf{X}, P_{3} \mathbf{X}$ are the three matrices deduced by taking the rows of $\left(\begin{array}{c}A \\ C\end{array}\right)$ three by three.

Let $Q_{1}, Q_{2}, Q_{3}$ the three matrices obtained by taking the rows of $\left(\begin{array}{c}B \\ D\end{array}\right)$ three by three. We want to check that $Q_{i}$ has maximal rank 3. This is obvious for $Q_{3}$ because the rows of $D$ are linearly independent. $Q_{1}$ has rank three too, because the first three rows of $F$ are linearly independent and $D$ is invertible. The last row of $B$ is the last row of $F$ times $D$, and so it is a linear combination of the last three rows of $D$ with non-zero coefficients. So, it is not a linear combination of the first two rows of $D$, again because $D$ has rank 5 . Hence, $Q_{2}$ has maximal rank 3, too, and the proof is complete.

\section{CRItical LOCI FOR PROJECTIVE RECONSTRUCTION OF LINES AND THEIR GEOMETRY}

In the previous sections, we have dealt with the reconstruction problem for sets of points from their images via three projections from $\mathbb{P}^{4}$ to $\mathbb{P}^{2}$. Moreover we have studied the critical loci for this problem.

A different reconstruction problem arises when one considers projections of lines in $\mathbb{P}^{3}$ instead of projections of points. This set-up has been considered and studied by various authors, in particular T.Buchanan [14] and S.J.Maybank [30]. Given a set of lines in $\mathbb{P}^{3}$ and $n$ projections of these lines to $\mathbb{P}^{2}$, T.Buchanan [14] shows that $n=3$ is the minimum $n$ such that it is possible to reconstruct the set from their images, up to projective transformations in $\mathbb{P}^{3}$. Of course, also in this context, there is a natural notion of critical locus, consisting of lines in $\mathbb{P}^{3}$.

Definition 6.1. A set of lines $\left\{\lambda_{j}\right\}, j=1, \ldots, N, N \gg 0$, in $\mathbb{P}^{3}$ is said to be a critical configuration for projective reconstruction of lines from three views if there exist two collections of $3 \times 4$ full-rank projection matrices $\varphi_{i}$ and $\psi_{i}, i=1,2,3$, and a set of $N$ lines $\left\{\mu_{j}\right\}$ in $\mathbb{P}^{3}$, non-projectively equivalent to $\left\{\lambda_{j}\right\}$, such that, for all $i$ and $j, \varphi_{i}\left(\lambda_{j}\right)=\psi_{i}\left(\mu_{j}\right)$, up to homography in the (dual) image planes. The two sets $\left\{\lambda_{j}\right\}$ and $\left\{\mu_{j}\right\}$ are called conjugate critical configurations, with associated conjugate matrices $\left\{\varphi_{i}\right\}$ and $\left\{\psi_{i}\right\}$.

In [14], the author, using geometrical arguments, shows that given two triples of projections from $\mathbb{P}^{3}$ to $\mathbb{P}^{2}$, the associated critical set for reconstruction via lines is a congruence $K$ of bi-degree $(3,6)$ and sectional genus 5 in the Grassmannian $\mathbb{G}(1,3)$ (see section 3 ).

In this section, via an algebraic approach, we compute the defining ideal of the critical locus for this reconstruction problem. We prove that the critical locus is the union of the above line congruence and of the three $\alpha$-planes in $\mathbb{G}(1,3)$ corresponding to the three centers of projection in $\mathbb{P}^{3}$.

To start, we denote by $\varphi_{i}: \mathbb{P}^{3} \rightarrow \mathbb{P}^{2}$ and $\psi_{i}: \mathbb{P}^{3} \rightarrow \mathbb{P}^{2}, i=1,2,3$, two triples of projections and we consider the associated critical locus for the reconstruction problem through lines considered by T.Buchanan [14]. Let $O_{i} \in \mathbb{P}^{3}$ (respectively, $O_{i}^{\prime} \in \mathbb{P}^{3}$ ) be the 
center of the projection $\varphi_{i}$ (resp. $\psi_{i}$ ). As in the following we are interested in the rows of the matrices $\psi_{1}, \psi_{2}, \psi_{3}$, we set

$$
\left(\begin{array}{c}
\psi_{1} \\
\psi_{2} \\
\psi_{3}
\end{array}\right)=\left(\begin{array}{c}
R_{1} \\
\vdots \\
R_{9}
\end{array}\right)
$$

where $R_{i}$ is a $1 \times 4$ matrix. As we consider general projections, we assume that the above matrix has rank 4.

As proved in [23], if we call $l$ a line in $\mathbb{P}^{3}$ and $l^{\prime}$ its image in $\mathbb{P}^{2}$ via a projection $\varphi=\left(\varphi_{i j}\right)$, the Plücker coordinates of $l$ are transformed into the Plücker coordinates of $l^{\prime}$ via

$$
\begin{aligned}
& \varphi \wedge \varphi= \\
& \left(\begin{array}{ll}
\left|\begin{array}{ll}
\varphi_{21} & \varphi_{22} \\
\varphi_{31} & \varphi_{32}
\end{array}\right||| \begin{array}{ll}
\varphi_{21} & \varphi_{23} \\
\varphi_{31} & \varphi_{33}
\end{array}|| \begin{array}{ll}
\varphi_{22} & \varphi_{23} \\
\varphi_{32} & \varphi_{33}
\end{array}|| \begin{array}{ll}
\varphi_{21} & \varphi_{24} \\
\varphi_{31} & \varphi_{34}
\end{array}|| \begin{array}{ll}
\varphi_{22} & \varphi_{24} \\
\varphi_{32} & \varphi_{34}
\end{array}|| \begin{array}{ll}
\varphi_{23} & \varphi_{24} \\
\varphi_{33} & \varphi_{34}
\end{array} \mid \\
\left|\begin{array}{ll}
\varphi_{31} & \varphi_{32} \\
\varphi_{11} & \varphi_{12}
\end{array}\right|\left|\begin{array}{ll}
\varphi_{31} & \varphi_{33} \\
\varphi_{11} & \varphi_{13}
\end{array}\right|\left|\begin{array}{ll}
\varphi_{32} & \varphi_{33} \\
\varphi_{12} & \varphi_{13}
\end{array}\right|\left|\begin{array}{ll}
\varphi_{31} & \varphi_{34} \\
\varphi_{11} & \varphi_{14}
\end{array}\right|\left|\begin{array}{ll}
\varphi_{32} & \varphi_{34} \\
\varphi_{12} & \varphi_{14}
\end{array}\right|\left|\begin{array}{ll}
\varphi_{33} & \varphi_{34} \\
\varphi_{13} & \varphi_{14}
\end{array}\right| \\
\left.\left|\begin{array}{ll}
\varphi_{11} & \varphi_{12} \\
\varphi_{21} & \varphi_{22}
\end{array}\right|\left|\begin{array}{ll}
\varphi_{11} & \varphi_{13} \\
\varphi_{21} & \varphi_{23}
\end{array}\right|\left|\begin{array}{ll}
\varphi_{12} & \varphi_{13} \\
\varphi_{22} & \varphi_{23}
\end{array}\right|\left|\begin{array}{ll}
\varphi_{11} & \varphi_{14} \\
\varphi_{21} & \varphi_{24}
\end{array}\right|\left|\begin{array}{ll}
\varphi_{12} & \varphi_{14} \\
\varphi_{22} & \varphi_{24}
\end{array}\right|\left|\begin{array}{ll}
\varphi_{13} & \varphi_{14} \\
\varphi_{23} & \varphi_{24}
\end{array}\right|\right)
\end{array}\right.
\end{aligned}
$$

Hence, we denote $\Phi_{i}=\varphi_{i} \wedge \varphi_{i}: \mathbb{P}^{5} \rightarrow \mathbb{P}^{2}$ and similarly $\Psi_{i}=\psi_{i} \wedge \psi_{i}$ both for $i=1,2,3$.

Remark 6.1. The center of the projection $\Phi_{i}$ is the 2 -plane in $\mathbb{P}^{5}$ defined by $\Phi_{i}(\mathbf{X})=\mathbf{0}$. They are contained in the Grassmannian $\mathbb{G}(1,3)$ and parameterize the lines of $\mathbb{P}^{3}$ through $O_{i}$, for every $i$, i.e. they are $\alpha$-planes.

In a natural way, then, we are induced to consider the reconstruction problem for points in $\mathbb{P}^{5}$ from three views, which turns out to be the minimum number needed for the reconstruction of a scene. Let $\mathbf{X}=\left(x_{0}, \ldots, x_{5}\right)^{T}$ be a point in $\mathbb{P}^{5}$. As done in section 5 while computing the critical locus for two triples of projections from $\mathbb{P}^{4}$ to $\mathbb{P}^{2}$, one has to consider the $9 \times 9$ matrix

$$
M=\left(\begin{array}{cccc}
\Phi_{1}(\mathbf{X}) & \mathbf{0} & \mathbf{0} & \Psi_{1} \\
\mathbf{0} & \Phi_{2}(\mathbf{X}) & \mathbf{0} & \Psi_{2} \\
\mathbf{0} & \mathbf{0} & \Phi_{3}(\mathbf{X}) & \Psi_{3}
\end{array}\right)
$$

where $\mathbf{0}$ is the $3 \times 1$ null matrix.

Remark 6.2. In the case of two triples of projections from $\mathbb{P}^{5}$ to $\mathbb{P}^{2}, \operatorname{det}(M)=0$ is the defining equation of the hypersurface that is critical for the reconstruction for points in this setting. Indeed, $\operatorname{det}(M)=0$ is equivalent to require that the linear system

$$
M\left(\begin{array}{c}
\lambda_{1} \\
\lambda_{2} \\
\lambda_{3} \\
\mathbf{Y}
\end{array}\right)=\mathbf{0}
$$

has a non-trivial solution such that $\mathbf{Y} \neq \mathbf{0}$.

The condition $\operatorname{det}(M)=0$ is not enough in our case to describe the critical locus since both $\mathbf{X}$ and $\mathbf{Y}$ are lines and so their coordinates must satisfy the equation of Klein hyperquadric. For $\mathbf{X}$ we have $x_{0} x_{5}-x_{1} x_{4}+x_{2} x_{3}=0$. The situation for $\mathbf{Y}$ is more involved. Indeed we have first to compute the coordinates of $\mathbf{Y}$ by solving suitable homogeneous linear systems, then to impose that such coordinates satisfy the Klein equation. 
If we denote by $M_{\hat{i}}$ the matrix obtained from $M$ by erasing the $i$-th row, the homogeneous linear system becomes

$$
M_{\hat{i}}\left(\begin{array}{c}
\lambda_{1} \\
\lambda_{2} \\
\lambda_{3} \\
\mathbf{Y}
\end{array}\right)=\mathbf{0} \quad 1 \leq i \leq 9 .
$$

It follows from Cramer's rule that

$$
y_{j}=(-1)^{1+j} \operatorname{det}\left(M_{\widehat{i, j+4}}\right), \quad j=0 \ldots 5,
$$

where $M_{\widehat{h, k}}$ is the matrix obtained from $M$ by erasing the $h$-th row and the $k$-th column. Now we have to impose that the computed $y_{0} \ldots y_{5}$ satisfy $y_{0} y_{5}-y_{1} y_{4}+y_{2} y_{3}=0$.

To simplify notations, we set

$$
\Phi_{i}(\mathbf{X})=\left(\begin{array}{c}
\Phi_{i 1} \\
\Phi_{i 2} \\
\Phi_{i 3}
\end{array}\right)
$$

that are the natural variables to construct the generators of the ideal of the critical locus $\mathcal{Y}$. In fact, while $\operatorname{det}(M)$ is a degree 3 form in the $\Phi_{i j}$ 's, irreducible in the general case, the form $y_{0} y_{5}-y_{1} y_{4}+y_{2} y_{3}$ has degree 6 and it is the product of three linear forms and a cubic form in the $\Phi_{i j}$ 's. To fix notations we denote by $g_{i}$ the cubic arising from the linear system associated to $M_{\hat{i}}$. For example, let us consider the linear system associated to $M_{\hat{1}}$. In this case, the linear forms are

$$
\begin{gathered}
\Phi_{21} \operatorname{det}\left(\begin{array}{c}
R_{7} \\
R_{8} \\
R_{9} \\
R_{4}
\end{array}\right)+\Phi_{22} \operatorname{det}\left(\begin{array}{l}
R_{7} \\
R_{8} \\
R_{9} \\
R_{5}
\end{array}\right)+\Phi_{23} \operatorname{det}\left(\begin{array}{l}
R_{7} \\
R_{8} \\
R_{9} \\
R_{6}
\end{array}\right)=0, \\
\Phi_{31} \operatorname{det}\left(\begin{array}{c}
R_{4} \\
R_{5} \\
R_{6} \\
R_{7}
\end{array}\right)+\Phi_{32} \operatorname{det}\left(\begin{array}{c}
R_{4} \\
R_{5} \\
R_{6} \\
R_{8}
\end{array}\right)+\Phi_{33} \operatorname{det}\left(\begin{array}{c}
R_{4} \\
R_{5} \\
R_{6} \\
R_{9}
\end{array}\right)=0, \\
\Phi_{12} b-\Phi_{13} a=0,
\end{gathered}
$$

where

$$
\begin{aligned}
a & =\left(\Psi_{1}\right)_{21} b_{12}-\left(\Psi_{1}\right)_{22} b_{13}+\left(\Psi_{1}\right)_{23} b_{23}+\left(\Psi_{1}\right)_{24} b_{14}-\left(\Psi_{1}\right)_{25} b_{24}+\left(\Psi_{1}\right)_{26} b_{34}, \\
b & =\left(\Psi_{1}\right)_{31} b_{12}-\left(\Psi_{1}\right)_{32} b_{13}+\left(\Psi_{1}\right)_{33} b_{23}+\left(\Psi_{1}\right)_{34} b_{14}-\left(\Psi_{1}\right)_{35} b_{24}+\left(\Psi_{1}\right)_{36} b_{34}
\end{aligned}
$$

and $\left(b_{12}, \ldots, b_{34}\right)$ are the Plücker coordinates of the line through the points

$$
\left(\operatorname{det}\left(\left(\psi_{2}\right)_{\hat{1}}\right), \operatorname{det}\left(\left(\psi_{2}\right)_{\hat{2}}\right), \operatorname{det}\left(\left(\psi_{2}\right)_{\hat{3}}\right), \operatorname{det}\left(\left(\psi_{2}\right)_{\hat{4}}\right)\right)
$$

and

$$
\left(\operatorname{det}\left(\left(\psi_{3}\right)_{\hat{1}}\right), \operatorname{det}\left(\left(\psi_{3}\right)_{\hat{2}}\right), \operatorname{det}\left(\left(\psi_{3}\right)_{\hat{3}}\right), \operatorname{det}\left(\left(\psi_{3}\right)_{\hat{4}}\right)\right),
$$

where, this time, $\hat{h}$ means that we erase the $h$-the column.

We have then proved the following:

Theorem 6.1. The defining ideal $I_{\mathcal{Y}}$ of the critical locus is generated by the Klein hyperquadric, $\operatorname{det}(M)$ and $g_{1} \ldots, g_{9}$.

Now we compute the minimal generators and free resolution of the ideal $I_{\mathcal{Y}}$. It is straightforward to check that

$$
g_{i}=\sum_{j, h, k}^{1,2,3} \Phi_{1 j} \Phi_{2 h} \Phi_{3 k} \operatorname{det}\left(\begin{array}{c}
R_{i} \\
R_{j} \\
R_{3+h} \\
R_{6+k}
\end{array}\right) \quad i=1, \ldots, 9
$$


while

$$
\operatorname{det}(M)=-\sum_{i, j, h}^{1,2,3} \Phi_{1 i} \Phi_{2 j} \Phi_{3 h} c_{i, 3+j, 6+h}
$$

where

$$
\begin{aligned}
\Phi_{i j h} & =\left(\begin{array}{c}
R_{i} \\
R_{j} \\
R_{h}
\end{array}\right) \text { and } \\
c_{i, j, h} & =\operatorname{det}\left(\begin{array}{cccc}
\operatorname{det}\left(\left(\Phi_{1}\right)_{\hat{1}}\right) & \operatorname{det}\left(\left(\Phi_{1}\right)_{\hat{2}}\right) & \operatorname{det}\left(\left(\Phi_{1}\right)_{\hat{3}}\right) & \operatorname{det}\left(\left(\Phi_{1}\right)_{\hat{4}}\right) \\
\operatorname{det}\left(\left(\Phi_{2}\right)_{\hat{1}}\right) & \operatorname{det}\left(\left(\Phi_{2}\right)_{\hat{2}}\right) & \operatorname{det}\left(\left(\Phi_{2}\right)_{\hat{3}}\right) & \operatorname{det}\left(\left(\Phi_{2}\right)_{\hat{4}}\right) \\
\operatorname{det}\left(\left(\Phi_{3}\right)_{\hat{1}}\right) & \operatorname{det}\left(\left(\Phi_{3}\right)_{\hat{2}}\right) & \operatorname{det}\left(\left(\Phi_{3}\right)_{\hat{3}}\right) & \operatorname{det}\left(\left(\Phi_{3}\right)_{\hat{4}}\right) \\
\operatorname{det}\left(\left(\Phi_{i j h}\right)_{\hat{1}}\right) & \operatorname{det}\left(\left(\Phi_{i j h}\right)_{\hat{2}}\right) & \operatorname{det}\left(\left(\Phi_{i j h}\right)_{\hat{3}}\right) & \operatorname{det}\left(\left(\Phi_{i j h}\right)_{\hat{4}}\right)
\end{array}\right) .
\end{aligned}
$$

Let $J$ be the ideal generated by $g_{1}, \ldots, g_{9}, \operatorname{det}(M)$. Then,

Proposition 6.1. The minimal free resolution of $J$ is

$$
0 \rightarrow T^{3}(-4) \rightarrow T^{4}(-3) \rightarrow J \rightarrow 0
$$

and so the scheme defined by $J$ is ACM of codimension 2 in $\mathbb{P}^{5}$.

Proof. Without loss of generality, we assume that $R_{1}, R_{2}, R_{4}, R_{7}$ are linearly independent, and so they are a basis of $\mathbb{C}^{4}$. Moreover, we set

$$
R_{i}=\alpha_{i 1} R_{1}+\alpha_{i 2} R_{2}+\alpha_{i 3} R_{4}+\alpha_{i 4} R_{7} \quad i=3,5,6,8,9
$$

and

$$
D=\operatorname{det}\left(\begin{array}{c}
R_{1} \\
R_{2} \\
R_{4} \\
R_{7}
\end{array}\right)
$$

We remark that $D$ is the coefficient of $\Phi_{12} \Phi_{21} \Phi_{31}$ in $g_{1}$.

First, we verify that $J$ is generated by $g_{1}, \ldots, g_{4}$. In fact, it holds

$$
\begin{aligned}
& g_{5}=-\frac{\alpha_{31} \alpha_{54}-\alpha_{34} \alpha_{51}}{\alpha_{34}} g_{1}-\frac{\alpha_{32} \alpha_{54}-\alpha_{34} \alpha_{52}}{\alpha_{34}} g_{2}+\frac{\alpha_{54}}{\alpha_{34}} g_{3}-\frac{\alpha_{33} \alpha_{54}-\alpha_{34} \alpha_{53}}{\alpha_{34}} g_{4} \\
& g_{6}=-\frac{\alpha_{31} \alpha_{64}-\alpha_{34} \alpha_{61}}{\alpha_{34}} g_{1}-\frac{\alpha_{32} \alpha_{64}-\alpha_{34} \alpha_{62}}{\alpha_{34}} g_{2}+\frac{\alpha_{64}}{\alpha_{34}} g_{3}-\frac{\alpha_{33} \alpha_{64}-\alpha_{34} \alpha_{63}}{\alpha_{34}} g_{4} \\
& g_{7}=-\frac{\alpha_{31}}{\alpha_{34}} g_{1}-\frac{\alpha_{32}}{\alpha_{34}} g_{2}+\frac{1}{\alpha_{34}} g_{3}-\frac{\alpha_{33}}{\alpha_{34}} g_{4} \\
& g_{8}=-\frac{\alpha_{31} \alpha_{84}-\alpha_{34} \alpha_{81}}{\alpha_{34}} g_{1}-\frac{\alpha_{32} \alpha_{84}-\alpha_{34} \alpha_{82}}{\alpha_{34}} g_{2}+\frac{\alpha_{84}}{\alpha_{34}} g_{3}-\frac{\alpha_{33} \alpha_{84}-\alpha_{34} \alpha_{83}}{\alpha_{34}} g_{4} \\
& g_{9}=-\frac{\alpha_{31} \alpha_{94}-\alpha_{34} \alpha_{91}}{\alpha_{34}} g_{1}-\frac{\alpha_{32} \alpha_{94}-\alpha_{34} \alpha_{92}}{\alpha_{34}} g_{2}+\frac{\alpha_{94}}{\alpha_{34}} g_{3}-\frac{\alpha_{33} \alpha_{94}-\alpha_{34} \alpha_{93}}{\alpha_{34}} g_{4} \\
& \operatorname{det}(M)=\frac{1}{\alpha_{34} D} c_{2,3,4} g_{1}-\frac{1}{\alpha_{34} D} c_{1,3,4} g_{2}+\frac{1}{\alpha_{34} D} c_{1,2,4} g_{3} .
\end{aligned}
$$

The equalities have to be checked by verifying that the coefficients of $\Phi_{1 i} \Phi_{2 j} \Phi_{3 h}$ are the same on both sides. It is very long, but straightforward. As example, we check some of them.

The first term we consider is $\Phi_{13} \Phi_{23} \Phi_{31}$ in the $g_{5}$ relation. The coefficient on the left side is equal to

$$
\operatorname{det}\left(\begin{array}{c}
R_{5} \\
R_{3} \\
R_{6} \\
R_{7}
\end{array}\right)=\operatorname{det}\left(\begin{array}{c}
\alpha_{51} R_{1}+\alpha_{52} R_{2}+\alpha_{53} R_{4} \\
\alpha_{31} R_{1}+\alpha_{32} R_{2}+\alpha_{33} R_{4} \\
\alpha_{61} R_{1}+\alpha_{62} R_{2}+\alpha_{63} R_{4} \\
R_{7}
\end{array}\right)=D \operatorname{det}\left(\begin{array}{ccc}
\alpha_{51} & \alpha_{52} & \alpha_{53} \\
\alpha_{31} & \alpha_{32} & \alpha_{33} \\
\alpha_{61} & \alpha_{62} & \alpha_{63}
\end{array}\right) .
$$


On the right, we have

$$
\begin{aligned}
& -\frac{\alpha_{31} \alpha_{54}-\alpha_{34} \alpha_{51}}{\alpha_{34}} \operatorname{det}\left(\begin{array}{c}
R_{1} \\
R_{3} \\
R_{6} \\
R_{7}
\end{array}\right)-\frac{\alpha_{32} \alpha_{54}-\alpha_{34} \alpha_{52}}{\alpha_{34}} \operatorname{det}\left(\begin{array}{c}
R_{2} \\
R_{3} \\
R_{6} \\
R_{7}
\end{array}\right)+\frac{\alpha_{54}}{\alpha_{34}} \operatorname{det}\left(\begin{array}{c}
R_{3} \\
R_{3} \\
R_{6} \\
R_{7}
\end{array}\right)- \\
& -\frac{\alpha_{33} \alpha_{54}-\alpha_{34} \alpha_{53}}{\alpha_{34}} \operatorname{det}\left(\begin{array}{c}
R_{4} \\
R_{3} \\
R_{6} \\
R_{7}
\end{array}\right)=-\frac{\alpha_{31} \alpha_{54}-\alpha_{34} \alpha_{51}}{\alpha_{34}} \operatorname{det}\left(\begin{array}{c}
R_{1} \\
\alpha_{32} R_{2}+\alpha_{33} R_{4} \\
\alpha_{62} R_{2}+\alpha_{63} R_{4} \\
R_{7}
\end{array}\right) \text { - } \\
& -\frac{\alpha_{32} \alpha_{54}-\alpha_{34} \alpha_{52}}{\alpha_{34}} \operatorname{det}\left(\begin{array}{c}
R_{2} \\
\alpha_{31} R_{1}+\alpha_{33} R_{4} \\
\alpha_{61} R_{1}+\alpha_{63} R_{4} \\
R_{7}
\end{array}\right)-\frac{\alpha_{33} \alpha_{54}-\alpha_{34} \alpha_{53}}{\alpha_{34}} \operatorname{det}\left(\begin{array}{c}
R_{4} \\
\alpha_{31} R_{1}+\alpha_{32} R_{2} \\
\alpha_{61} R_{1}+\alpha_{62} R_{2} \\
R_{7}
\end{array}\right)= \\
& =D\left\{-\frac{\alpha_{54}}{\alpha_{34}}\left(\alpha_{31} \operatorname{det}\left(\begin{array}{ll}
\alpha_{32} & \alpha_{33} \\
\alpha_{62} & \alpha_{63}
\end{array}\right)-\alpha_{32} \operatorname{det}\left(\begin{array}{ll}
\alpha_{31} & \alpha_{33} \\
\alpha_{61} & \alpha_{63}
\end{array}\right)+\alpha_{33} \operatorname{det}\left(\begin{array}{ll}
\alpha_{31} & \alpha_{32} \\
\alpha_{61} & \alpha_{62}
\end{array}\right)\right)+\right. \\
& \left.+\left(\alpha_{51} \operatorname{det}\left(\begin{array}{ll}
\alpha_{32} & \alpha_{33} \\
\alpha_{62} & \alpha_{63}
\end{array}\right)-\alpha_{52} \operatorname{det}\left(\begin{array}{ll}
\alpha_{31} & \alpha_{33} \\
\alpha_{61} & \alpha_{63}
\end{array}\right)+\alpha_{53} \operatorname{det}\left(\begin{array}{ll}
\alpha_{31} & \alpha_{32} \\
\alpha_{61} & \alpha_{62}
\end{array}\right)\right)\right\}= \\
& =D\left\{-\frac{\alpha_{54}}{\alpha_{34}} \operatorname{det}\left(\begin{array}{lll}
\alpha_{31} & \alpha_{32} & \alpha_{33} \\
\alpha_{31} & \alpha_{32} & \alpha_{33} \\
\alpha_{61} & \alpha_{62} & \alpha_{63}
\end{array}\right)+\operatorname{det}\left(\begin{array}{lll}
\alpha_{51} & \alpha_{52} & \alpha_{53} \\
\alpha_{31} & \alpha_{32} & \alpha_{33} \\
\alpha_{61} & \alpha_{62} & \alpha_{63}
\end{array}\right)\right\}
\end{aligned}
$$

and so the equality holds.

Now, we check the equality of the coefficients of $\Phi_{11} \Phi_{21} \Phi_{31}$ in the $\operatorname{det}(M)$ relation. On the left side, we have $-c_{147}$. On the right side, we have

$$
\begin{aligned}
& +\frac{1}{\alpha_{34} D} c_{234} \operatorname{det}\left(\begin{array}{c}
R_{1} \\
R_{1} \\
R_{4} \\
R_{7}
\end{array}\right)-\frac{1}{\alpha_{34} D} c_{134} \operatorname{det}\left(\begin{array}{c}
R_{2} \\
R_{1} \\
R_{4} \\
R_{7}
\end{array}\right)+\frac{1}{\alpha_{34} D} \operatorname{det}\left(\begin{array}{c}
R_{3} \\
R_{1} \\
R_{4} \\
R_{7}
\end{array}\right)= \\
& =\frac{1}{\alpha_{34}} c_{134}+\frac{1}{\alpha_{34} D} \operatorname{det}\left(\begin{array}{c}
\alpha_{32} R_{2} \\
R_{1} \\
R_{4} \\
R_{7}
\end{array}\right)=\frac{1}{\alpha_{34}} \operatorname{det}\left(\begin{array}{ccc}
\operatorname{det}\left(\left(\Phi_{1}\right)_{\hat{1}}\right) & \ldots \\
\operatorname{det}\left(\left(\Phi_{2}\right)_{\hat{1}}\right) & \ldots \\
\operatorname{det}\left(\left(\Phi_{3}\right)_{\hat{1}}\right) & \ldots \\
\operatorname{det}\left(\left(\Phi_{134}-\alpha_{32} \Phi_{124}\right)_{\hat{1}}\right) & \ldots
\end{array}\right)= \\
& =\frac{1}{\alpha_{34}} \operatorname{det}\left(\begin{array}{cc}
\operatorname{det}\left(\left(\Phi_{1}\right)_{\hat{1}}\right) & \ldots \\
\operatorname{det}\left(\left(\Phi_{2}\right)_{\hat{1}}\right) & \ldots \\
\operatorname{det}\left(\left(\Phi_{3}\right)_{\hat{1}}\right) & \ldots \\
\operatorname{det}\left(\left(\alpha_{34} \Phi_{174}\right)_{\hat{1}}\right) & \ldots
\end{array}\right)=-c_{147}
\end{aligned}
$$

and so the equality holds also in this case.

As second step, we check that the minors with sign of the following matrix $N$ are equal to $g_{1}, \ldots, g_{4}$. 


$$
\left(\begin{array}{cccc}
\frac{D}{\alpha_{34}} \Phi_{11} & -\left|\begin{array}{cc}
\alpha_{31} & \alpha_{34} \\
\alpha_{51} & \alpha_{54}
\end{array}\right| \Phi_{22}-\left|\begin{array}{cc}
\alpha_{31} & \alpha_{34} \\
\alpha_{61} & \alpha_{64}
\end{array}\right| \Phi_{23} & -\alpha_{31} \Phi_{31}-\left|\begin{array}{cc}
\alpha_{31} & \alpha_{34} \\
\alpha_{81} & \alpha_{84}
\end{array}\right| \Phi_{32}- \\
\frac{D}{\alpha_{34}} \Phi_{12} & -\left|\begin{array}{cc}
\alpha_{32} & \alpha_{34} \\
\alpha_{52} & \alpha_{54}
\end{array}\right| \Phi_{22}-\left|\begin{array}{cc}
\alpha_{32} & \alpha_{34} \\
\alpha_{62} & \alpha_{64}
\end{array}\right| \Phi_{23} & -\alpha_{32} \Phi_{31}-\left|\begin{array}{cc}
\alpha_{32} & \alpha_{34} \\
\alpha_{82} & \alpha_{84}
\end{array}\right| \Phi_{32}- \\
\frac{D}{\alpha_{34}} \Phi_{13} & -\left|\begin{array}{cc}
\alpha_{32} & \alpha_{34} \\
\alpha_{92} & \alpha_{94}
\end{array}\right| \Phi_{33} \\
0 & \alpha_{54} \Phi_{22}+\alpha_{64} \Phi_{23} & \Phi_{31}+\alpha_{84} \Phi_{32}+\alpha_{94} \Phi_{33} \\
\alpha_{34} \Phi_{21}-\left|\begin{array}{cc}
\alpha_{33} & \alpha_{34} \\
\alpha_{53} & \alpha_{54}
\end{array}\right| \Phi_{22} & -\alpha_{33} \Phi_{31}-\left|\begin{array}{cc}
\alpha_{33} & \alpha_{34} \\
\alpha_{83} & \alpha_{84}
\end{array}\right| \Phi_{32}- \\
-\left|\begin{array}{ccc}
\alpha_{33} & \alpha_{34} \\
\alpha_{63} & \alpha_{64}
\end{array}\right| \Phi_{23} & -\left|\begin{array}{cc}
\alpha_{33} & \alpha_{34} \\
\alpha_{93} & \alpha_{94}
\end{array}\right| \Phi_{33}
\end{array}\right) .
$$

Once again, the check is straightforward. For example, let us consider the minor obtained by erasing the last row of $N$ and let us compute the coefficient of $\Phi_{11} \Phi_{22} \Phi_{33}$ in the minor. It holds

$$
\frac{D}{\alpha_{34}}\left\{-\alpha_{94}\left|\begin{array}{cc}
\alpha_{32} & \alpha_{34} \\
\alpha_{52} & \alpha_{54}
\end{array}\right|+\alpha_{54}\left|\begin{array}{cc}
\alpha_{32} & \alpha_{34} \\
\alpha_{92} & \alpha_{94}
\end{array}\right|\right\}=\frac{D}{\alpha_{34}} \alpha_{34}\left|\begin{array}{cc}
\alpha_{52} & \alpha_{54} \\
\alpha_{92} & \alpha_{94}
\end{array}\right|=\operatorname{det}\left(\begin{array}{c}
R_{4} \\
R_{1} \\
R_{5} \\
R_{9}
\end{array}\right)
$$

and so we get $\operatorname{det}\left(N_{\hat{4}}\right)=g_{4}$. With analogous computations, we $\operatorname{get} \operatorname{det}\left(N_{\hat{1}}\right)=-g_{1}$, $\operatorname{det}\left(N_{\hat{2}}\right)=g_{2}$, and $\operatorname{det}\left(N_{\hat{3}}\right)=-g_{3}$. As $g_{1}, \ldots, g_{4}$ are irreducible for general choices, we get the claim by Hilbert-Burch Theorem. Moreover, $N$ represents the map $T^{3}(-4) \rightarrow T^{4}(-3)$ in the resolution of $J$.

Remark 6.3. The scheme defined by $J$ in $\mathbb{P}^{5}$ is a lifting of a Bordiga surface in $\mathbb{P}^{4}$ in the following sense. Let $\ell$ be a general linear form in $T$. Hence there is an isomorphism between $R$ and $T / \ell$. As $J$ is ACM the minimal free resolution of $J$ remains exact over $T / \ell$ and so $(J+\ell) / \ell$ defines a Bordiga surface in $\mathbb{P}^{4}=\operatorname{Proj}(R)$. Moreover, the matrices $N$ and $N_{\mathcal{X}}$ of the two minimal free resolutions have the same properties: the three columns span linear spaces of codimension 3 , in position $(4,1)$ there is a zero, and the element in position $(3,2)$ is a linear combination of the ones in positions $(1,2)$ and $(2,2)$. The main difference is that the linear spaces spanned by the columns are pairwise skew in the case of the Bordiga surface, while every couple of them has a point in common in the case of $J$, as the codimension 2 linear spaces are $\alpha$-planes in this case, and so the intersection of two of them is the point corresponding to the line through the centers of the two nets of lines.

Now we can describe the geometry of the critical locus, starting from its defining ideal, and Buchanan's result.

Theorem 6.2. The critical locus for the reconstruction problem for a pair of three projections $\varphi_{i}, \psi_{j}, i, j=1,2,3$, of lines from $\mathbb{P}^{3}$ to $\mathbb{P}^{2}$ is the union of a line congruence of bi-degree $(3,6)$ and sectional genus 5 and the three $\alpha$-planes associated to the projection centers of the $\varphi_{i}$ 's. Moreover, each $\alpha$-plane intersects the line congruence along a degree 3 plane curve.

Proof. The scheme defined by $J$ is irreducible for general choices, and so the Klein hyperquadric is regular with respect to it. As the defining ideal of the critical locus $\mathcal{Y}$ is 
$I_{\mathcal{Y}}=J+\left(x_{0} x_{5}-x_{1} x_{4}+x_{2} x_{3}\right) T$, then its minimal free resolution is

$$
0 \rightarrow T^{3}(-6) \rightarrow \underset{\substack{T^{4}(-5) \\ T^{3}(-4)}}{\rightarrow} \underset{\substack{\oplus \\ T^{4}(-3)}}{\rightarrow} \rightarrow I_{\mathcal{Y}} \rightarrow 0
$$

and so the Hilbert polynomial of $\mathcal{Y}$ is $p_{\mathcal{Y}}(t)=\left(12 t^{2}-8 t+8\right) / 2$. Hence, $\mathcal{Y}$ is a surface in $\mathbb{P}^{5}$ of degree 12. It is an easy check to verify that the centers $\alpha_{1}, \alpha_{2}, \alpha_{3}$ of the projections $\Phi_{1}, \Phi_{2}, \Phi_{3}$ are the $\alpha$-planes associated to the centers of $\varphi_{1}, \varphi_{2}, \varphi_{3}$ respectively and are contained in $\mathcal{Y}$, as every maximal minor of $N$ is a combination of $\Phi_{i 1}, \ldots, \Phi_{i 3}$, for $i=$ $1,2,3$. Moreover, we know that a line congruence $K$ of bi-degree $(3,6)$ and sectional genus 5 is contained in the critical locus. Then, by degree argument, $\mathcal{Y}=K \cup \alpha_{1} \cup \alpha_{2} \cup \alpha_{3}$. By using the same argument as in Proposition 3.3, we get that each $\alpha_{i}$ intersects $K$ along a degree 3 plane curve, as claimed.

\section{A BRidge Between the two RECONSTRUCtion PROBlems}

In this section, we want to show that the two reconstruction problems considered in the previous sections are related each other, in the following sense. Given two triples of projections from $\mathbb{P}^{4}$ to $\mathbb{P}^{2}$, and the corresponding critical locus $\mathcal{X} \subset \mathbb{P}^{4}$, it is possible to determine two triples of projections from $\mathbb{P}^{3}$ to $\mathbb{P}^{2}$ in such a way that the critical locus for the reconstruction problem for lines is the union of three suitable $\alpha$-planes and the image of $\mathcal{X}$ in $\mathbb{G}(1,3)$ via the rational map $\theta: \mathbb{P}^{4} \rightarrow \mathbb{G}(1,3)$ quoted in (7). Furthermore, also the converse holds. Now we describe various steps to get the $\varphi_{i}$ and $\psi_{j}$ from the $P_{i}$ and $Q_{j}$.

(1) Let $P_{i}, Q_{j}: \mathbb{P}^{4} \rightarrow \mathbb{P}^{2}$ be projections, with $i, j=1,2,3$. The critical locus $\mathcal{X} \subset$ $\mathbb{P}^{4}$ for the reconstruction problem for points has been studied in section 5 . In particular, we proved that $\mathcal{X}$ is a Bordiga surface, and we computed the $4 \times 3$ matrix $N_{\mathcal{X}}$ whose columns generate the first syzygy module of $I_{\mathcal{X}}$ from the $9 \times 8$ matrix $M$ whose maximal minors generate the same ideal $I_{\mathcal{X}}$.

(2) As explained at the end of section 2, a degree 4 rational normal curve $\Gamma \subset \mathcal{X}$ can be obtained by taking the maximal minors $q_{0}, \ldots, q_{5}$ of $N_{\mathcal{X}} M_{L}$ where $M_{L}$ is a general $3 \times 2$ matrix of maximal rank. To fix the order, $q_{0}=\operatorname{det}\left(N_{\mathcal{X}} M_{L}\right)_{\widehat{34}}, q_{1}=$ $\operatorname{det}\left(N_{\mathcal{X}} M_{L}\right)_{\widehat{24}}, q_{2}=\operatorname{det}\left(N_{\mathcal{X}} M_{L}\right)_{\widehat{14}}, q_{3}=\operatorname{det}\left(N_{\mathcal{X}} M_{L}\right)_{\widehat{23}}, q_{4}=\operatorname{det}\left(N_{\mathcal{X}} M_{L}\right)_{\widehat{13}}, q_{5}=$ $\operatorname{det}\left(N_{\mathcal{X}} M_{L}\right)_{\widehat{12}}$, where, as usual, .. means that the corresponding rows are omitted.

(3) Following [40], we define the map $\theta: T=H_{*}^{0}\left(\mathcal{O}_{\mathbb{P}^{5}}\right) \rightarrow R=H_{*}^{0}\left(\mathcal{O}_{\mathbb{P}^{4}}\right)$ by setting $\theta\left(x_{i}\right)=q_{i}, i=0, \ldots, 5$. Then, $\theta^{-1}\left(I_{\mathcal{X}}\right)=I_{K}$ where $K$ is a line congruence of bi-degree $(3,6)$ and sectional genus 5 as the ones studied in section 3 .

(4) From equation (3), we get $N_{Z}$ from $N_{\mathcal{X}}$ where the columns of $N_{Z}$ generate the first syzygy module of $I_{Z}$ in $S=H_{*}^{0}\left(\mathcal{O}_{\mathbb{P}^{2}}\right), Z$ being a set of 10 points in general position, among which there are $p_{1}(1: 0: 0), p_{2}(0: 1: 0), p_{3}(0: 0: 1)$. We remark that $\mathcal{X}$ is the image of the embedding of $\widetilde{\mathbb{P}}^{2}$, blow-up of $\mathbb{P}^{2}$ in $Z$, via the linear system $\left|4 \pi^{*} L-E_{1}-\cdots-E_{10}\right|$.

(5) Let $C_{i} \subset \mathbb{P}^{2}$ be the plane cubic curve containing $Z \backslash\left\{p_{i}\right\}$, with $i=1,2,3$. The equation of $C_{i}$ is the only degree 3 generator of $I_{Z}: I_{p_{i}}$. Let $C_{i}^{\prime} \subset \mathcal{X}$ be the image of $C_{i}$ and $C_{i}^{\prime \prime} \subset K$ be the image of $C_{i}^{\prime}$. The saturated ideal that defines $C_{i}^{\prime \prime}$ is $\theta^{-1}\left(I_{C_{i}^{\prime}}\right)$ and so we can compute the plane $\alpha_{i}$ spanned by $C_{i}^{\prime \prime}$. The critical locus we are looking for is $\mathcal{Y}=K \cup \alpha_{1} \cup \alpha_{2} \cup \alpha_{3}$.

(6) The last map in the minimal free resolution of $I_{\mathcal{Y}}$ is $T^{3}(-6) \rightarrow T^{4}(-5) \oplus T^{3}(-4)$. Let $N_{\mathcal{Y}}$ be the matrix that represents $T^{3}(-6) \rightarrow T^{4}(-5)$ : the maximal minors of this matrix define the ideal $J$ and so we can recover the projection matrices $\Phi_{i}, \Psi_{j}$ from it, $i, j=1,2,3$, as explained in Proposition 6.1. Hence, it is possible to compute the projections $\varphi_{i}, \psi_{j}: \mathbb{P}^{3} \rightarrow \mathbb{P}^{2}$ we want to construct. 
Now, we give an algorithm that summarizes the previous discussion, and that can be easily implemented in Singular [15], for example.

Input: projection matrices $P_{1}, P_{2}, P_{3}, Q_{1}, Q_{2}, Q_{3}$.

Output: projection matrices $\varphi_{1}, \varphi_{2}, \varphi_{3}, \psi_{1}, \psi_{2}, \psi_{3}$.

(1.1) Given the $5 \times 1$ matrix $V Y=\left[y_{0}, \ldots, y_{4}\right]$, compute $P_{1} \cdot V Y, P_{2} \cdot V Y, P_{3} \cdot V Y$ and the $9 \times 8$ matrix $M$.

(1.2) Compute the matrix $N_{\mathcal{X}}=A-B D^{-1} C$ as in section 5, and the ideal $I_{\mathcal{X}}$ of the maximal minors of $N_{\mathcal{X}}$.

(2) Choose a general $3 \times 2$ matrix $M_{L}$ of numbers, compute $M_{\Gamma}=N_{\mathcal{X}} M_{L}$, and its maximal minors $q_{0}, \ldots, q_{5}$ in the above order.

(3) Define the map $\theta$ by setting $\theta\left(x_{i}\right)=q_{i}, i=0, \ldots, 5$, and compute $I_{K}=\theta^{-1}\left(I_{\mathcal{X}}\right)$.

(4.1) Given the $3 \times 1$ matrix $V Z=\left[z_{0}, z_{1}, z_{2}\right]$, compute the matrix $N_{Z}$ from $N_{\mathcal{X}}$ according to equation (3).

(4.2) Compute $g_{0}, \ldots, g_{4}$, maximal minors of $N_{Z}$ with alternating signs, and set $I_{Z}$ the ideal they generate.

(4.3) Define the map $\pi$ by setting $\pi\left(y_{i}\right)=g_{i}, i=0, \ldots, 4$.

(5.1) Compute $F_{1}=\left(I_{Z}:\left\langle z_{1}, z_{2}\right\rangle\right)_{3}, F_{2}=\left(I_{Z}:\left\langle z_{0}, z_{2}\right\rangle\right)_{3}, F_{3}=\left(I_{Z}:\left\langle z_{0}, z_{1}\right\rangle\right)_{3}$ and $I_{\alpha_{i}}=\left(\theta^{-1} \circ \pi^{-1}\left(F_{i}\right)\right)_{1}, i=1,2,3$, where $(\ldots)_{j}$ means the degree $j$ part of the ideal in parenthesis.

(5.2) Compute $I_{\mathcal{Y}}=I_{K} \cap\left(\bigcap_{i=1,2,3} I_{\alpha_{i}}\right)$.

(6.1) Compute the third matrix in the minimal free resolution of $I_{\mathcal{Y}}$ and take the submatrix with linear entries.

(6.2) Put this matrix in the form of the proof of Proposition 6.1.

(6.3) Compute matrices $\Phi_{i}, \Psi_{j}, 1 \leq i, j \leq 3$, from the last matrix, and the associated projection matrices $\varphi_{i}, \psi_{j}$.

Now, we describe the converse construction.

(1) Given the projections $\varphi_{i}, \psi_{j}: \mathbb{P}^{3} \rightarrow \mathbb{P}^{2}$, we can compute the maps $\Phi_{i}, \Psi_{j}$ and the critical locus $\mathcal{Y} \subset \mathbb{G}(1,3)$ for the reconstruction problem for projections of lines, as explained in section 6 . Moreover, we compute the line congruence $K$ and the three $\alpha$-planes that are the irreducible components of $\mathcal{Y}$.

(2) As explained in section 3, we compute a Veronese surface $V \subset \mathbb{G}(1,3)$ such that $K \cup V$ is an arithmetically Gorenstein surface of degree 13 and $K \cap V$ is a degree 10 arithmetically Gorenstein curve.

(3) Let $\theta^{-1}: R \rightarrow T_{\mathbb{G}}$ be the map associated to $K \cap V$, where $T_{\mathbb{G}}=T /\left\langle x_{0} x_{5}-x_{1} x_{4}+\right.$ $\left.x_{2} x_{3}\right\rangle$ is the coordinate ring of the Grassmannian $\mathbb{G}(1,3)$. Then, $\theta^{-1}\left(I_{K}\right)=I_{\mathcal{X}}$ where $\mathcal{X}$ is a Bordiga surface.

(4) From Theorem 5.1, we get projections $P_{i}, Q_{j}: \mathbb{P}^{4} \rightarrow \mathbb{P}^{2}, 1 \leq i, j \leq 3$, such that $\mathcal{X}$ is the associated critical locus for the reconstruction problem.

As before, we give the corresponding algorithm. It can be implemented in Singular [15].

Input: projection matrices $\varphi_{1}, \varphi_{2}, \varphi_{3}, \psi_{1}, \psi_{2}, \psi_{3}$.

Output: projection matrices $P_{1}, P_{2}, P_{3}, Q_{1}, Q_{2}, Q_{3}$.

(1.1) Compute $\Phi_{i}, \Psi_{j}, 1 \leq i, j \leq 3$, as in section 6 .

(1.2) Compute the critical locus $\mathcal{Y}$ for the reconstruction problem for lines, and its irreducible components.

(2) In the line congruence $K$ contained in $\mathcal{Y}$, compute a degree 10 arithmetically Gorenstein curve $C=K \cap V$ whose ideal is generated by 5 quadrics $q_{0}, \ldots, q_{4}$ in addition to the Klein hyperquadric $x_{0} x_{5}-x_{1} x_{4}+x_{2} x_{3}$.

(3) Define $\theta^{-1}: R \rightarrow T_{\mathbb{G}}$ as $\theta^{-1}\left(y_{i}\right)=q_{i}, i=0, \ldots, 4$, and compute $\theta^{-1}\left(I_{K}\right)=I_{\mathcal{X}}$. 
(4) Compute the generators of the first syzygy module $N_{\mathcal{X}}$ of $I_{\mathcal{X}}$ and compute a basis from which it is possible to reconstruct the projections $P_{i}, Q_{j}: \mathbb{P}^{4} \rightarrow \mathbb{P}^{2}, 1 \leq i, j \leq$ 3 , we are looking for.

\section{A COMPUter SESSION CONCERNING THE LINE CONGRUENCE}

In this section, we present a computer session in Singular in which, staring from the ideal of a general line congruence $K$ of bi-degree $(3,6)$ and sectional genus 5 , we compute a degree 13 arithmetically Gorenstein surface $S$ such that $S=K \cup V$ where $V$ is a Veronese surface, and $K \cap V$ is a degree 10 arithmetically Gorenstien curve that allows us to compute the map $\theta^{-1}: \mathbb{G}(1,3) \rightarrow \mathbb{P}^{4}$.

Input: ideal $I_{K}$ of a general line congruence.

Output: ideals $I_{S}$ and $I_{C}$ where $S=K \cup V, C=K \cap V$.

ring $r=0,(x(0 . .5), a(1 . .268)), \mathrm{dp}$;

ideal $i_{k}$; \ This ideal has to be given as input. The first generator is the Klein hyperquadric.

option(redSB);

ideal $k_{1}=\operatorname{std}\left(i_{k}\right) ; \quad \|$ reduced standard basis of $i_{k}$

\In the following lines, we compute general polynomials that will become a reduced standard basis of $i_{s}$

matrix mon3[1][46] $=x(5)^{3}, x(4) * x(5)^{2}, x(3) * x(5)^{2}, x(2) * x(5)^{2}, x(1) * x(5)^{2}, x(0) *$ $x(5)^{2}, x(4)^{2} * x(5), x(3) * x(4) * x(5), x(2) * x(4) * x(5), x(1) * x(4) * x(5), x(0) * x(4) *$ $x(5), x(3)^{2} * x(5), x(1) * x(3) * x(5), x(0) * x(3) * x(5), x(2)^{2} * x(5), x(1) * x(2) * x(5), x(0) *$ $x(2) * x(5), x(1)^{2} * x(5), x(0) * x(1) * x(5), x(0)^{2} * x(5), x(4)^{3}, x(3) * x(4)^{2}, x(2) * x(4)^{2}, x(1) *$ $x(4)^{2}, x(0) * x(4)^{2}, x(3)^{2} * x(4), x(1) * x(3) * x(4), x(0) * x(3) * x(4), x(2)^{2} * x(4), x(1) * x(2) *$ $x(4), x(0) * x(2) * x(4), x(1)^{2} * x(4), x(0) * x(1) * x(4), x(0)^{2} * x(4), x(3)^{3}, x(1) * x(3)^{2}, x(0) *$ $x(3)^{2}, x(1)^{2} * x(3), x(0) * x(1) * x(3), x(0)^{2} * x(3), x(2)^{3}, x(1) * x(2)^{2}, x(0) * x(2)^{2}, x(1)^{2} *$ $x(2), x(0) * x(1) * x(2), x(1)^{3}$;

matrix $c p 1[46][1]=a(1 . .46)$;

poly $p_{1}=(\operatorname{mon} 3 * c p 1)[1,1]+x(0)^{3}$;

matrix $c p 2[46][1]=a(47 . .92)$;

poly $p_{2}=(\operatorname{mon} 3 * c p 2)[1,1]+x(0)^{2} * x(1)$;

matrix $c p 3[46][1]=a(93 . .138)$;

poly $p_{3}=(\operatorname{mon} 3 * c p 3)[1,1]+x(0) * x(1)^{2}$;

matrix $c p 4[45][1]=a(139 . .183)$;

poly $p_{4}=(\operatorname{submat}(m o n 3, \operatorname{intvec}(1), \operatorname{intvec}(1 . .45)) * c p 4)[1,1]+x(0)^{2} * x(2)$;

matrix mon $4[1][85]=x(5)^{4}, x(4) * x(5)^{3}, x(3) * x(5)^{3}, x(2) * x(5)^{3}, x(1) * x(5)^{3}, x(0) *$ $x(5)^{3}, x(4)^{2} * x(5)^{2}, x(3) * x(4) * x(5)^{2}, x(2) * x(4) * x(5)^{2}, x(1) * x(4) * x(5)^{2}, x(0) * x(4) *$ $x(5)^{2}, x(3)^{2} * x(5)^{2}, x(1) * x(3) * x(5)^{2}, x(0) * x(3) * x(5)^{2}, x(2)^{2} * x(5)^{2}, x(1) * x(2) * x(5)^{2}, x(0) *$ $x(2) * x(5)^{2}, x(1)^{2} * x(5)^{2}, x(0) * x(1) * x(5)^{2}, x(0)^{2} * x(5)^{2}, x(4)^{3} * x(5), x(3) * x(4)^{2} * x(5), x(2) *$ $x(4)^{2} * x(5), x(1) * x(4)^{2} * x(5), x(0) * x(4)^{2} * x(5), x(3)^{2} * x(4) * x(5), x(1) * x(3) * x(4) *$ $x(5), x(0) * x(3) * x(4) * x(5), x(2)^{2} * x(4) * x(5), x(1) * x(2) * x(4) * x(5), x(0) * x(2) * x(4) *$ $x(5), x(1)^{2} * x(4) * x(5), x(0) * x(1) * x(4) * x(5), x(0)^{2} * x(4) * x(5), x(3)^{3} * x(5), x(1) * x(3)^{2} *$ $x(5), x(0) * x(3)^{2} * x(5), x(1)^{2} * x(3) * x(5), x(0) * x(1) * x(3) * x(5), x(0)^{2} * x(3) * x(5), x(2)^{3} *$ $x(5), x(1) * x(2)^{2} * x(5), x(0) * x(2)^{2} * x(5), x(1)^{2} * x(2) * x(5), x(0) * x(1) * x(2) * x(5), x(1)^{3} *$ $x(5), x(4)^{4}+a(231) * x(3) * x(4)^{3}, x(2) * x(4)^{3}, x(1) * x(4)^{3}, x(0) * x(4)^{3}, x(3)^{2} * x(4)^{2}, x(1) *$ $x(3) * x(4)^{2}, x(0) * x(3) * x(4)^{2}, x(2)^{2} * x(4)^{2}, x(1) * x(2) * x(4)^{2}, x(0) * x(2) * x(4)^{2}, x(1)^{2} *$ $x(4)^{2}, x(0) * x(1) * x(4)^{2}, x(0)^{2} * x(4)^{2}, x(3)^{3} * x(4), x(1) * x(3)^{2} * x(4), x(0) * x(3)^{2} * x(4), x(1)^{2} *$ $x(3) * x(4), x(0) * x(1) * x(3) * x(4), x(0)^{2} * x(3) * x(4), x(2)^{3} * x(4), x(1) * x(2)^{2} * x(4), x(0) *$ $x(2)^{2} * x(4), x(1)^{2} * x(2) * x(4), x(0) * x(1) * x(2) * x(4), x(1)^{3} * x(4), x(3)^{4}, x(1) * x(3)^{3}, x(0) *$ $x(3)^{3}, x(1)^{2} * x(3)^{2}, x(0) * x(1) * x(3)^{2}, x(0)^{2} * x(3)^{2}, x(1)^{3} * x(3), x(2)^{4}, x(1) * x(2)^{3}, x(0) *$ $x(2)^{3}, x(1)^{2} * x(2)^{2}, x(0) * x(1) * x(2)^{2}, x(1)^{3} * x(2)$; 
matrix $c p 5[85][1]=a(184 . .268)$;

poly $p_{5}=(\operatorname{mon} 4 * c p 5)[1,1]+x(1)^{4}$;

ideal rel; $\quad \backslash$ This ideal will contain the relations among the $a(j)$ 's

poly $s p ; \quad \backslash$ the following are local variables

int $i$;

int $j$;

int $k$;

int $f l$;

ideal $m x=x(0 . .5)$;

matrix $m x 3[1][56]=\operatorname{std}\left(m x^{3}\right) ; \quad \|$ monomials of degree 3

matrix $m x 4[1][126]=\operatorname{std}\left(m x^{4}\right) ; \quad \backslash \backslash$ monomials of degree 4

matrix $l p 1[1][8]=\operatorname{std}\left(\operatorname{lead}\left(k_{1}\right)\right) ; \quad \|$ leading monomials of $k_{1}$

for $(i=1 ; i<=8 ; i=i+1)\left\{k_{1}[i]=k_{1}[i] /\right.$ leadcoef $\left.\left(k_{1}[i]\right) ;\right\} ; \quad \backslash$ normalized reduced standard basis of $k_{1}$;

\I we compute the normal form of $p 1$ with respect to $k_{1}$ and we collect the coefficients of the monomials in the ideal rel

$s p=p 1$

for $(j=56 ; j>=1 ; j=j-1)\{$

$f l=0$;

$k=0$;

while $((k<8)$ and $(f l==0))\{$

$k=k+1$;

$\operatorname{if}(\operatorname{gcd}(l p 1[1, k], m x 3[1, j])==\operatorname{lp} 1[1, k])\{f l=1 ;\}\} ;$

if $(f l==0)\{$

if $(s p<>$ reduce $(s p, \operatorname{std}(m x 3[1, j])))\{$

$r e l=r e l,(s p-\operatorname{reduce}(s p, \operatorname{std}(m x 3[1, j]))) / m x 3[1, j] ;\} ;$

$s p=\operatorname{reduce}(s p, \operatorname{std}(m x 3[1, j])) ;\}$;

if $(f l==1)\{$

$\left.\left.s p=s p-((s p-\operatorname{reduce}(s p, \operatorname{std}(m x 3[1, j]))) / \operatorname{lp} 1[1, k]) * k_{1}[k] ;\right\} ;\right\} ;$

$\|$ repeat verbatim the above computation for $s p=p 2, p 3, p 4$ and we collect the coefficients of the monomials in the same ideal rel

$s p=p 2$

:

$\|$ we compute the normal form of $p 5$ with respect to $k_{1}$ and we collect the coefficients of the monomials in the same ideal rel

$s p=p 5$

for $(j=126 ; j>=1 ; j=j-1)\{$

$f l=0$;

$k=0$;

while $((k<8)$ and $(f l==0))\{$

$k=k+1$;

$\operatorname{if}(\operatorname{gcd}(l p 1[1, k], m x 4[1, j])==l p 1[1, k])\{f l=1 ;\}\} ;$

if $(f l==0)\{$

$\operatorname{if}(s p<>\operatorname{reduce}(s p, \operatorname{std}(m x 4[1, j])))\{$

$r e l=r e l,(s p-\operatorname{reduce}(s p, \operatorname{std}(m x 4[1, j]))) / m x 4[1, j] ;\}$;

$s p=\operatorname{reduce}(s p, \operatorname{std}(m x 4[1, j])) ;\}$;

if $(f l==1)\{$

$\left.\left.s p=s p-((s p-\operatorname{reduce}(s p, \operatorname{std}(m x 4[1, j]))) / \operatorname{lp} 1[1, k]) * k_{1}[k] ;\right\} ;\right\}$

$\|$ we compute a standard basis of $r e l$, and we reduce $p 1, \ldots, p 5$ modulo it. After this step, they belong to $i_{k}$

$r e l=\operatorname{std}(r e l)$;

poly $p 11=\operatorname{reduce}(p 1, r e l)$; 
poly $p 21=\operatorname{reduce}(p 2, r e l)$;

poly $p 31=\operatorname{reduce}(p 3, \mathrm{rel})$;

poly $p 41=\operatorname{reduce}(p 4, r e l)$;

poly $p 51=\operatorname{reduce}(p 5, r e l)$;

\ we run Buchberger's algorithm of the set $\left\{i_{k}[1], p 11, \ldots, p 51\right\}$ and we compute the coefficient in such a way that they form a Gröbner basis. The relations are stored in rel

kill rel;

ideal rel;

matrix $\operatorname{lp}[1][6]=x(2) * x(3), x(0)^{3}, x(0)^{2} * x(1), x(0) * x(1)^{2}, x(0)^{2} * x(2), x(1)^{4}$;

matrix $p p[1][6]=x(2) * x(3)-x(1) * x(4)+x(0) * x(5), p 11, p 21, p 31, p 41, p 51$;

intmat $b[9][2]=1,5,2,3,2,5,3,4,3,5,2,4,4,5,4,6,3,6$;

for $(i=1 ; i<=5 ; i=i+1)\{$

$s p=(\operatorname{lp}[1, b[i, 2]] * p p[1, b[i, 1]]-\operatorname{lp}[1, b[i, 1]] * p p[1, b[i, 2]])$;

$s p=s p / \operatorname{gcd}(\operatorname{lp}[1, b[i, 2]], \operatorname{lp}[1, b[i, 1]])$;

for $(j=126 ; j>=1 ; j=j-1)\{$

$f l=0$;

$k=0$;

while $((k<6) \operatorname{and}(f l==0))\{$

$k=k+1$

if $(\operatorname{gcd}(\operatorname{lp}[1, k], m x 4[1, j])==\operatorname{lp}[1, k])\{f l=1 ;\}\}$;

if $(f l==0)\{$

if $(s p<>\operatorname{reduce}(s p, \operatorname{std}(m x 4[1, j])))\{$

$r e l=r e l,(s p-\operatorname{reduce}(s p, \operatorname{std}(m x 4[1, j]))) / m x 4[1, j] ;\} ;$

$s p=\operatorname{reduce}(s p, \operatorname{std}(m x 4[1, j])) ;\}$;

$\operatorname{if}(f l==1)\{$

$s p=s p-((s p-\operatorname{reduce}(s p, \operatorname{std}(m x 4[1, j]))) / l p[1, k]) * p p[1, k] ;\} ;\} ;\} ;$

\I we choose randomly the values of $a(182), a(183)$ and compute a standard basis of $r e l$ : in such a way, every coefficient has a numerical value that we substitute in $p 11, \ldots, p 51$

ideal rel1 $=a(182)-\operatorname{random}(-5,5), a(183)-\operatorname{random}(-5,5)$, rel;

rel1 $=\operatorname{std}($ rel1 $)$

poly $p 12=\operatorname{reduce}(p 11$, rel 1$)$;

poly $p 22=\operatorname{reduce}(p 21$, rel 1$)$;

poly $p 32=\operatorname{reduce}(p 31$, rel 1$)$;

poly $p 42=\operatorname{reduce}(p 41$, rel 1$)$

poly $p 52=\operatorname{reduce}(p 51$, rel 1$)$;

ideal $i_{s}=i_{k}[1], p 12, p 22, p 32, p 42, p 52$;

ideal $i_{v}=$ quotient $\left(i_{s}, i_{k}\right)$;

ideal $i_{c}=i_{v}, i_{k}$;

\section{REFERENCES}

[1] E. Arrondo, and I. Sols On conguences of lines in projective space. Mémoires de la S.M.F. $2^{a}$ série, tome 50, (1992).

[2] K. Åström and F. Kahl Ambiguous Configurations for the $1 D$ Structure and Motion Problem. Journal of Mathematical Imaging and Vision, 18:191-203, 2003.

[3] M. Bertolini, G. Besana, and C. Turrini. Instability of projective reconstruction of dynamic scenes near critical configurations. In Proceedings of the International Conference on Computer Vision, $I C C V, 2007$.

[4] M. Bertolini, G. Besana, and C. Turrini. Instability of projective reconstruction from 1-view near critical configurations in higher dimensions. In Algebra Geometry and their Interactions, volume 448 of Contemporary Mathematics, 2007.

[5] M. Bertolini, G. Besana, and C. Turrini. Reconstruction of some segmented and dynamic scenes: trifocal tensor in $\mathbb{P}^{4}$, theoretical set up for critical loci and instability. In Advances in Visual Computing, Proceedings of the International Symposium on Visual Computing, ISVC 2008, Part II, volume 5359. Springer Verlag, 2008. 
[6] M. Bertolini, G. Besana, and C. Turrini. Applications Of Multiview Tensors In Higher Dimensions in "Tensors in Image Processing and Computer Vision", Editors: Aja-Fernndez, S., de Luis Garcia, R., Tao, D., Li, X., Advances in Pattern Recognition. 2009.

[7] M. Bertolini, G. Besana, and C. Turrini. Critical loci for projective reconstruction from multiple views in higher dimension: A comprehensive theoretical approach. Linear Algebra and its Applications, 469(2015), 335-363.

[8] M. Bertolini, G. Besana, and C. Turrini. Generalized Fundamental Matrices as Grassmann Tensors Annali di Matematica Pura ed Applicata, n.2, 196(2017), 539-553.

[9] M. Bertolini, G. Besana, R. Notari and C. Turrini. Provisional title: Critical loci for 3-view reconstruction in $\mathbb{P}^{4}$ : the degenerate cases and instability. In progress.

[10] M. Bertolini and L. Magri. Critical hypersurfaces and instability for reconstruction of scenes in high dimensional projective spaces. Preprint, 2017.

[11] M. Bertolini and C. Turrini. Critical configurations for 1-view in projections from $\mathbb{P}^{k} \rightarrow \mathbb{P}^{2}$. Journal of Mathematical Imaging and Vision, 2007.

[12] G. Bordiga. La superficie del $6^{\circ}$ ordine con 10 rette, nello spazio $R_{4}$ e le sue proiezioni nello spazio ordinario. Mem.Accad.Lincei, (4) 3 (1887), 182-203.

[13] T. Buchanan. The twisted cubic and camera calibration. Comput. Vision Graphics Image Process., 42(1):130-132, 1988.

[14] T. Buchanan. On the critical set for photogrammetric reconstruction using line tokens in $\mathbb{P}_{\mathbb{C}}^{3}$. Geometriae Dedicata, 44:223-232, 1992.

[15] W. Decker, G.M. Greuel, G. Pfister, H. Schönemann. Singular 4-1-0 - A computer algebra system for polynomial computations. http://www.singular.uni-kl.de (2016).

[16] I.V. Dolgachev. Classical Algebraic Geometry. A modern view. Cambridge University Press, first edition, 2012.

[17] D. Eisenbud. Commutative Algebra. Graduate Texts in Mathematics 150. Springer, New York, third edition, 1999.

[18] G. Ellingsrud. Sur le schéma de Hilbert des variétés de codimension 2 dans $\mathbb{P}^{e}$ à cône de CohenMacaulay. Ann. Sci. École Norm. Sup. (4) 8 (1975), no. 4, 423-431.

[19] R.I. Hartley. Ambiguous configurations for 3-view projective reconstruction. In European Conference on Computer Vision, pages I: 922-935, 2000.

[20] R.I. Hartley and F. Kahl. Critical configurations for projective reconstruction from multiple views. International Journal of Computer Vision, 71(1):5-47, 2007.

[21] R.I. Hartley and F. Schaffalitzky. Reconstruction from projections using Grassmann tensors. In Proceedings of the 8th European Conference on Computer Vision, Prague, Czech Republic, LNCS. Springer, 2004.

[22] R. Hartley and R. Vidal. The multibody trifocal tensor: Motion segmentation from 3 perspective views. In Conference on Computer Vision and Pattern Recognition, 2004.

[23] R.I. Hartley and A. Zisserman. Multiple View Geometry in Computer Vision. Cambridge University Press, 2004.

[24] F. Kahl, R. Hartley, and K. Åström. Critical configurations for n-view projective reconstruction. In IEEE Computer Society Conference on Computer Vision and Pattern Recognition, pages II:158-163, 2001.

[25] J.O. Kleppe, J.C. Migliore, R. Miró-Roig, U. Nagel, and C. Peterson. Gorenstein Liaison, Complete Intersection Liaison Invariants and Onubstructedness. Mem. Amer. Math. Soc., n. 732, Vol. 154, (2001).

[26] J. Kollár, K.E. Smith, and A. Corti. Rational and nearly rational varieties, In Cambridge Studies in Advanced Mathematics. Cambridge University Press, Cambridge, 2004.

[27] J. Krames. Zur ermittlung eines objectes aus zwei perspectiven (ein beitrag zur theorie der "gefhählichen Örter"). Monatsh. Math. Phys., 49:327-354, 1940.

[28] Kun Huang, R. Fossum, and Yi Ma. Generalized rank conditions in multiple view geometry with applications to dynamical scenes. In European Conference on Computer Vision, 2002.

[29] S.J. Maybank. Theory of Reconstruction from Image Motion. Springer-Verlag New York, Inc., Secaucus, NJ, USA, 1992.

[30] S.J. Maybank. The critical line congruence for reconstruction from three images. AAECC, 6:89-113, 1995.

[31] R. Notari and M.L. Spreafico A stratification of Hilbert schemes by initial ideals and applications. Manuscripta Math., 101, (2000), 429-448.

[32] G. Ottaviani. Varietà proiettive di codimensione piccola. INdAM - Aracne, 1995.

[33] J.G. Semple and L. Roth. Introduction to Algebraic Geometry. Oxford at the Clarendon Press, 1949.

[34] J. Shamash. The Poincaré series of a local ring. J. Algebra, 12:453470, 1969. 
[35] A. Shashua and S.J. Maybank. Degenerate $n$ point configurations of three views: Do critical surfaces exist? TR 96-19, Hebrew University, 1996.

[36] R. Vidal and Yi Ma. A unified algebraic approach to 2-d and 3-d motion segmentation and estimation. In Journal of Mathematical Imaging and Vision, 2006.

[37] R. Vidal, Yi Ma, S. Soatto, and S. Sastry. Two-view multibody structure from motion. In International Journal of Computer Vision, 2006.

[38] Xiaodong Fan and R. Vidal. The space of multibody fundamental matrices: Rank, geometry and projection. In Dynamical Vision, volume 4358 of Lecture Notes in Computer Science, 2007.

[39] L. Wolf and A. Shashua. On projection matrices $\mathbb{P}^{k} \rightarrow \mathbb{P}^{2}, k=3, \ldots, 6$ and their applications in computer vision. In International Journal of Computer Vision, 2002.

[40] S. Verra. Smooth surfaces of degree 9 in $\mathbb{G}(1,3)$. Manuscripta Mathematica, 62:416-435, 1988.

E-mail address: marina.bertolini@unimi.it

E-mail address: roberto.notari@polimi.it

E-mail address: cristina.turrini@unimi.it

Dipartimento di Matematica "F. Enriques", Università degli Studi di Milano, Via SalDiNi 50, 20133 Milano, ItaLy

Dipartimento di Matematica "F.Brioschi", Politecnico di Milano, Piazza Leonardo da Vinci 32, 20133 MiLANO 